TRANSACTIONS OF THE

AMERICAN MATHEMATICAL SOCIETY

Volume 352 Number 6 , Pages $2445-2477$

S 0002-9947(00)02594-0

Article electronically published on February 24, 2000

\title{
A PROBABILISTIC APPROACH TO POSITIVE HARMONIC FUNCTIONS IN A SLAB WITH ALTERNATING DIRICHLET AND NEUMANN BOUNDARY CONDITIONS
}

\author{
ROSS G. PINSKY
}

AbStract. Let $\Omega=R^{d} \times(-1,1), d \geq 2$, be a $d+1$ dimensional slab. Denote points $z \in R^{d+1}$ by $z=(r, \theta, y)$, where $(r, \theta) \in[0, \infty) \times S^{d-1}$ and $y \in R$. Denoting the boundary of the slab by $\Gamma=\partial \Omega$, let

$$
\Gamma_{D}=\left\{z=(r, \theta, y) \in \Gamma: r \in \bigcup_{n=1}^{\infty}\left(a_{n}, b_{n}\right)\right\}
$$

where $\left\{\left(a_{n}, b_{n}\right)\right\}_{n=1}^{\infty}$ is an ordered sequence of intervals on the right half line (that is, $a_{n+1}>b_{n}$ ). Assume that the lengths of the intervals are bounded and that the spaces between consecutive intervals are bounded and bounded away from zero. Let $\Gamma_{N}=\Gamma-\bar{\Gamma}_{D}$. Let $C_{B}\left(\Omega ; \Gamma_{D}, \Gamma_{N}\right)$ and $C_{P}\left(\Omega ; \Gamma_{D}, \Gamma_{N}\right)$ denote respectively the cone of bounded, positive harmonic functions in $\Omega$ and the cone of positive harmonic functions in $\Omega$ which satisfy the Dirichlet boundary condition on $\Gamma_{D}$ and the Neumann boundary condition on $\Gamma_{N}$.

Letting $\rho_{n} \equiv b_{n}-a_{n}$, the main result of this paper, under a modest assumption on the sequence $\left\{\rho_{n}\right\}$, may be summarized as follows when $d \geq 3$ :

1. If $\sum_{n=1}^{\infty} \frac{n}{\left|\log \rho_{n}\right|}<\infty$, then $\mathcal{C}_{B}\left(\Omega, \Gamma_{D}, \Gamma_{N}\right)$ and $\mathcal{C}_{P}\left(\Omega, \Gamma_{D}, \Gamma_{N}\right)$ are both one-dimensional (as in the case of the Neumann boundary condition on the entire boundary). In particular, this occurs if $\rho_{n}=\exp \left(-n^{l}\right)$ with $l>2$.

2. If $\sum_{n=1}^{\infty} \frac{n}{\left|\log \rho_{n}\right|}=\infty$ and $\sum_{n=1}^{\infty} \frac{\left|\log \rho_{n}\right|^{\frac{1}{2}}}{n^{2}}=\infty$, then $\mathcal{C}_{B}\left(\Omega, \Gamma_{D}, \Gamma_{N}\right)=$ $\varnothing$ and $\mathcal{C}_{P}\left(\Omega, \Gamma_{D}, \Gamma_{N}\right)$ is one-dimensional. In particular, this occurs if $\rho_{n}=$ $\exp \left(-n^{2}\right)$.

3. If $\sum_{n=1}^{\infty} \frac{\left|\log \rho_{n}\right|^{\frac{1}{2}}}{n^{2}}<\infty$, then $\mathcal{C}_{B}\left(\Omega, \Gamma_{D}, \Gamma_{N}\right)=\varnothing$ and the set of minimal elements generating $\mathcal{C}_{P}\left(\Omega, \Gamma_{D}, \Gamma_{N}\right)$ is isomorphic to $S^{d-1}$ (as in the case of the Dirichlet boundary condition on the entire boundary). In particular, this occurs if $\rho_{n}=\exp \left(-n^{l}\right)$ with $0 \leq l<2$.

When $d=2, \mathcal{C}_{B}\left(\Omega, \Gamma_{D}, \Gamma_{N}\right)=\varnothing$ as soon as there is at least one interval of Dirichlet boundary condition. The dichotomy for $\mathcal{C}_{P}\left(\Omega, \Gamma_{D}, \Gamma_{N}\right)$ is as above.

Let $\Omega=R^{d} \times(-1,1), d \geq 2$, be a $d+1$ dimensional slab. Denoting the boundary of the slab by $\Gamma=\partial \Omega$, let $\Gamma_{D}$ be a relatively open subset of $\Gamma$, let $\Gamma_{N}=\Gamma-\bar{\Gamma}_{D}$, and assume that $\Gamma_{D}$ is sufficiently nice so that $\Gamma-\Gamma_{N}-\Gamma_{D} \subset R^{d+1}$ is polar for $d+1$-dimensional Brownian motion. Consider a $d+1$-dimensional Brownian

Received by the editors January 4, 1999.

1991 Mathematics Subject Classification. Primary 35J05, 31C35, 31B05, 60J50.

Key words and phrases. Positive harmonic functions, Martin boundary, Dirichlet boundary condition, Neumann boundary condition, harmonic measure.

This research was done while the author was on sabbatical at the Courant Institute of Mathematical Sciences. 
motion in $\Omega$ which is killed at $\Gamma_{D}$ and normally reflected at $\Gamma_{N}$. No condition is imposed on $\Gamma-\Gamma_{D}-\Gamma_{N}$, since with probability one, the Brownian motion will never reach this part of the boundary. The generator of this Brownian motion with reflection and killing is $\frac{1}{2} \Delta$ in $\Omega$ with the Dirichlet boundary condition on $\Gamma_{D}$ and the Neumann boundary condition on $\Gamma_{N}$. In this paper, we use probabilistic tools to study the cone $C_{B}\left(\Omega ; \Gamma_{D}, \Gamma_{N}\right)$ of bounded, positive harmonic functions in $\Omega$ and the cone $C_{P}\left(\Omega ; \Gamma_{D}, \Gamma_{N}\right)$ of positive harmonic functions in $\Omega$ which satisfy the Dirichlet boundary condition on $\Gamma_{D}$ and the Neumann boundary condition on $\Gamma_{N}$.

In order to motivate this problem, we note what occurs in the case that $\Gamma_{D}=\varnothing$ and in the case that $\Gamma_{D}=\Gamma$. In the case that $\Gamma_{D}=\varnothing$, that is, the case in which reflection occurs along the entire boundary, $C_{B}(\Omega, \varnothing, \Gamma)=C_{P}(\Omega, \varnothing, \Gamma)=$ \{ the positive constants $\}$; in particular, these cones are one dimensional. On the other hand, in the case $\Gamma_{D}=\Gamma$, that is, the case in which killing occurs along the entire boundary, the cone $C_{B}(\Omega, \Gamma, \varnothing)$ is empty while the set of minimal elements generating the cone $C_{P}(\Omega, \Gamma, \varnothing)$ is isomorphic to $S^{d-1}$. (In fact, the cone is generated by the functions $\left\{e^{\frac{\pi}{2} \theta \cdot x} \cos \frac{\pi y}{2}\right\}_{\theta \in S^{d-1}}$, where $x \in R^{d}$ and $y \in[-1,1]$.) The fact that $C_{B}(\Omega, \Gamma, \varnothing)$ is empty follows from (1.2) below. The proofs of the claims concerning $C_{P}(\Omega, \varnothing, \Gamma)$ and $C_{P}(\Omega, \Gamma, \varnothing)$ are sketched at the end of this section, and a reference is provided.

Denote points $z \in \Omega$ by $z=(r, \theta, y)$, where $(r, \theta) \in[0, \infty) \times S^{d-1}$ denote polar coordinates in $R^{d}$ and $y \in(-1,1)$. In this paper, we will assume that $\Gamma_{D}$ has the form

$$
\Gamma_{D}=\left\{z=(r, \theta, y) \in \Gamma: r \in \bigcup_{n=1}^{\infty}\left(a_{n}, b_{n}\right)\right\},
$$

where $\left\{\left(a_{n}, b_{n}\right)\right\}_{n=1}^{\infty}$ is an ordered sequence of intervals on the right half line

(that is, $b_{n}<a_{n+1}$ ) satisfying

$\sup _{n}\left(b_{n}-a_{n}\right)<\infty$ and

$0<\inf _{n}\left(a_{n+1}-b_{n}\right) \leq \sup _{n}\left(a_{n+1}-b_{n}\right)<\infty$.

In other words, we assume that $\Gamma_{D}$ is composed of a countable number of uniformly bounded radial intervals and that the distance between neighboring intervals is bounded and bounded away from zero.

Before turning to the details, we describe briefly the nature of our results. It turns out that there are two threshold sizes for $\Gamma_{D}$. Assume first that $d \geq 3$. If $\Gamma_{D}$ is sufficiently small, then $C_{B}\left(\Omega, \Gamma_{D}, \Gamma_{N}\right)$ and $C_{P}\left(\Omega, \Gamma_{D}, \Gamma_{N}\right)$ will both be one dimensional, as in the case that $\Gamma_{D}=\varnothing$ (although, of course, the elements of these cones will no longer be the positive constants). In particular, this will occur if $b_{n}-a_{n}=\exp \left(-n^{l}\right)$ with $l>2$. If $\Gamma_{D}$ is sufficiently large, then, as in the case that $\Gamma_{D}=\Gamma, C_{B}\left(\Omega, \Gamma_{D}, \Gamma_{N}\right)$ will be empty while the set of minimal elements generating the cone $C_{P}\left(\Omega, \Gamma_{D}, \Gamma_{N}\right)$ will be isomorphic to $S^{d-1}$. In particular, this will occur if $b_{n}-a_{n}=\exp \left(-n^{l}\right)$ with $l<2$. In between these two thresholds lies a narrow range where $C_{B}\left(\Omega, \Gamma_{D}, \Gamma_{N}\right)$ will be empty and $C_{P}\left(\Omega, \Gamma_{D}, \Gamma_{N}\right)$ will be one-dimensional. In particular, this will occur if $b_{n}-a_{n}=\exp \left(-n^{2}\right)$. When $d=2, C_{B}\left(\Omega, \Gamma_{D}, \Gamma_{N}\right)=\varnothing$ for all $\Gamma_{D}$ as above, and more generally, for any $\Gamma_{0}$ which contains a relatively open set. The smaller of the two threshold sizes is now at $\Gamma_{D}=\varnothing$ and the larger one is as in the case $d \geq 3$. 
We now discuss the probabilistic representation of $C_{B}\left(\Omega, \Gamma_{D}, \Gamma_{N}\right)$ and of $C_{P}\left(\Omega, \Gamma_{D}, \Gamma_{N}\right)$. Before starting to define processes, we note that the canonical path notation $z(t)=(r(t), \theta(t), y(t))$, along with the filtration $\mathcal{F}_{t}=\sigma(z(s), 0 \leq s \leq t)$, will be used throughout; processes will be distinguished by the measure. Throughout this paper, $P_{z}$ and $E_{z}$ will denote probabilities and expectations for a Brownian motion in $\Omega$ starting from $z \in \Omega$, with reflection along the entire boundary.

Let $\sigma=\inf \left\{t \geq 0: z(t) \in \Gamma_{D}\right\}$. Then it is easy to demonstrate that

$$
\mathcal{C}_{B}\left(\Omega, \Gamma_{D}, \Gamma_{N}\right)=\varnothing \text { if and only if } P_{z}(\sigma<\infty)=1,
$$

for some, or equivalently, for any $z \in \Omega$.

To see this, first note that $u_{0}(z) \equiv P_{z}(\sigma=\infty)$ is harmonic and thus, by the strong maximum principle, either $P_{z}(\sigma<\infty)=1$ for all $z \in \Omega$ or $P_{z}(\sigma<\infty)<1$ for all $z \in \Omega$. Thus, in the latter case, $u_{0} \in \mathcal{C}_{B}\left(\Omega, \Gamma_{D}, \Gamma_{N}\right)$. For the other direction, assume that $u \in \mathcal{C}_{B}\left(\Omega, \Gamma_{D}, \Gamma_{N}\right)$. Then $u(z(t \wedge \sigma))$ is a bounded martingale. Under the assumption that $P_{z}(\sigma<\infty)=1$, we arrive at the contradiction $u(z)=\lim _{t \rightarrow \infty} E_{z} u(z(t \wedge \sigma))=E_{z} u(z(\sigma))=0$.

Before considering the probabilistic representation for $\mathcal{C}_{P}\left(\Omega, \Gamma_{D}, \Gamma_{N}\right)$, we recall its analytic representation via the Martin boundary at infinity for the operator $\frac{1}{2} \Delta$ on $\Omega$ with the mixed Dirichlet and Neumann boundary conditions. Let $G(z, w)$ denote the Green's function for $\frac{1}{2} \Delta$ in $\Omega$ with the mixed Dirichlet and Neumann boundary conditions. (The probabilistic representation of $G(z, w)$ is as the density in $w$ of the Green's measure $G(z, A) \equiv E_{z} \int_{0}^{\sigma} I_{A}(z(t)) d t$.) Fix $z_{0} \in \Omega$ and define

$$
k(z, w)=\frac{G(z, w)}{G\left(z_{0}, w\right)} .
$$

For any sequence $\left\{w_{n}\right\} \subset \Omega$ satisfying $\lim _{n \rightarrow \infty}\left|w_{n}\right|=\infty$, there exists a subsequence $\left\{w_{n}^{\prime}\right\}$ such that $u(z) \equiv \lim _{n \rightarrow \infty} k\left(z, w_{n}^{\prime}\right)$ exists and $u \in \mathcal{C}_{P}\left(\Omega ; \Gamma_{D}, \Gamma_{N}\right)$. A sequence $\left\{w_{n}\right\}$ for which $\lim _{n \rightarrow \infty} k\left(z, w_{n}\right) \in \mathcal{C}_{P}\left(\Omega, \Gamma_{D}, \Gamma_{N}\right)$ is called a Martin sequence, and two such sequences giving rise to the same element in $\mathcal{C}_{P}\left(\Omega, \Gamma_{D}, \Gamma_{N}\right)$ are defined to be equivalent. The collection of equivalence classes is called the Martin boundary at infinity. Denote the Martin boundary at infinity by $\Lambda$, denote elements in $\Lambda$ by $\zeta$, and denote the positive harmonic function in $\mathcal{C}_{P}\left(\Omega, \Gamma_{D}, \Gamma_{N}\right)$ corresponding to $\zeta$ by $u(\cdot ; \zeta)$. A function $u \in \mathcal{C}_{P}\left(\Omega, \Gamma_{D}, \Gamma_{N}\right)$ is called minimal if whenever $v \in$ $\mathcal{C}_{P}\left(\Omega, \Gamma_{D}, \Gamma_{N}\right)$ and $v \leq u$, then in fact $v=c u$ for some $c>0$. The minimal Martin boundary at infinity is defined by $\Lambda_{0} \equiv\{\zeta \in \Lambda: u(\cdot ; \zeta)$ is minimal $\}$. The Martin representation theorem states that, for each $u \in \mathcal{C}_{P}\left(\Omega, \Gamma_{D}, \Gamma_{N}\right)$, there exists a unique finite measure $\nu_{u}$ on $\Lambda_{0}$ such that $u(z)=\int_{\Lambda_{0}} u(z ; \zeta) \nu_{u}(d \zeta)$.

We now turn to the probabilistic representation for $\mathcal{C}_{P}\left(\Omega, \Gamma_{D}, \Gamma_{N}\right)$. Actually, more precisely, we give a probabilistic representation for the Martin boundary at infinity for the operator $\frac{1}{2} \Delta$ in $\Omega$ with the mixed Dirichlet and Neumann boundary conditions. Let $\Omega_{0}=\{(r, \theta, y) \in \Omega: r<1\}$ denote the unit cylinder in $\Omega$. Define the stopping times $\tau_{r}=\inf \{t \geq 0: r(t)=r\}$. Note that starting from $\Omega-\Omega_{0}$, the first hitting time of $\bar{\Omega}_{0}$ is $\tau_{1}$. Let

$$
h(z)=P_{z}\left(\tau_{1}<\sigma\right)
$$

Then $\frac{1}{2} \Delta h=0$ in $\Omega-\Omega_{0}$, and $h$ satisfies the Dirichlet boundary condition on $\Gamma_{D}-\partial \Omega_{0}$ and the Neumann boundary condition on $\Gamma_{N}-\partial \Omega_{0}$. The Brownian motion with reflection along the entire boundary, conditioned on the event 
$\left\{\tau_{1}<\sigma\right\}$, and run until $\tau_{1}$, is generated by the $h$-transformed Laplacian $\frac{1}{2} \Delta^{h}$ (defined by $\Delta^{h} f=\frac{1}{h} \Delta(h f)$ ) with the Neumann boundary condition on $\Gamma_{N}-\partial \Omega_{0}$. (No boundary conditioned is imposed on $\Gamma_{D}-\partial \Omega_{0}$ because it is inaccessible under $\frac{1}{2} \Delta^{h}$.) Writing out $\Delta^{h}$ explicitly (note that $h$ does not depend on $\theta$ ), we have

$$
\frac{1}{2} \Delta^{h}=\left(\frac{1}{2} \frac{\partial^{2}}{\partial r^{2}}+\frac{d-1}{2 r} \frac{\partial}{\partial r}+\frac{h_{r}}{h} \frac{\partial}{\partial r}\right)+\left(\frac{1}{2} \frac{\partial^{2}}{\partial y^{2}}+\frac{h_{y}}{h} \frac{\partial}{\partial y}\right)+\frac{1}{2 r^{2}} \Delta_{S^{d-1}},
$$

where $\Delta_{S^{d-1}}$ denotes the Laplace-Beltrami operator on $S^{d-1}$. Denote by $P_{z}^{h}$ and $E_{z}^{h}$ the probability and corresponding expectation for the conditioned process starting from $z \in \Omega-\Omega_{0}$. Define the conditioned exterior harmonic measure on $S^{d-1} \times[-1,1]$ by

$$
\begin{aligned}
\mu_{z}(d \theta, d y) & =P_{z}\left(\theta\left(\tau_{1}\right) \in d \theta, y\left(\tau_{1}\right) \in d y \mid \tau_{1}<\sigma\right) \\
& =P_{z}^{h}\left(\theta\left(\tau_{1}\right) \in d \theta, y\left(\tau_{1}\right) \in d y\right) .
\end{aligned}
$$

The connection between the Martin boundary and the conditioned exterior harmonic measure is direct: a sequence $\left\{w_{n}\right\}$ satisfying $\lim _{n \rightarrow \infty}\left|w_{n}\right|=\infty$ is a Martin sequence if and only if the sequence $\left\{\mu_{w_{n}}(d \theta, d y)\right\}$ of probability measures converges weakly as $n \rightarrow \infty$. Furthermore, two such sequences are equivalent Martin sequences if and only if they give rise to the same limiting measure. (See [2], [3] which deal with the Dirichlet boundary condition; the introduction of mixed boundary conditions causes no problem.)

We now state two assumptions on the lengths of the intervals which form the boundary set $\Gamma_{D}$.

Assumption 1. $\rho_{n} \equiv b_{n}-a_{n}$ is "essentially decreasing"; that is, there exists an integer $Q>1$ such that

$$
\rho_{m} \leq \rho_{n}, \text { for } m \geq Q n .
$$

Assumption 2. Let $\rho_{n} \equiv b_{n}-a_{n}$.

$$
\text { If } \liminf _{n \rightarrow \infty} \frac{\left|\log \rho_{n}\right|}{n^{2}}=0, \text { then } \limsup _{n \rightarrow \infty} \frac{\left|\log \rho_{n}\right|}{n^{2}}<\infty .
$$

Under these two assumptions, our results give succinct necessary and sufficient conditions. Without these assumptions, the statements of the results are a bit cumbersome and fall just short of being necessary and sufficient. Thus, for the sake of clarity of exposition, we present Theorem 1 under Assumption 1 and Theorem 2 under Assumptions 1 and 2, and then go on to the more general Theorems $1^{\prime}$ and $2^{\prime}$.

Theorem 1. Let $\Gamma_{D}$ be as in (1.1), let $\rho_{n}=b_{n}-a_{n}$, and let Assumption 1 hold. i. If $d \geq 3$ and

$$
\sum_{n=1}^{\infty} \frac{n}{\left|\log \rho_{n}\right|}<\infty
$$

then

$$
P_{z}(\sigma<\infty)<1
$$

ii. If $d=2$, or if $d \geq 3$ and

$$
\sum_{n=1}^{\infty} \frac{n}{\left|\log \rho_{n}\right|}=\infty,
$$


then

$$
P_{z}(\sigma<\infty)=1
$$

In case (i), $\mathcal{C}_{B}\left(\Omega, \Gamma_{D}, \Gamma_{N}\right)$ is one dimensional, and in case (ii), $\mathcal{C}_{B}\left(\Omega, \Gamma_{D}, \Gamma_{N}\right)=\varnothing$.

Theorem 2. Let $\Gamma_{D}$ be as in (1.1), let $\rho_{n}=b_{n}-a_{n}$, and let Assumption 1 and 2 hold.

i. If

$$
\sum_{n=1}^{\infty} \frac{\left|\log \rho_{n}\right|^{\frac{1}{2}}}{n^{2}}<\infty,
$$

then for any sequence $\left\{r_{n}, \theta_{n}, y_{n}\right\}$ satisfying $\lim _{n \rightarrow \infty} r_{n}=\infty$,

$$
\lim _{n \rightarrow \infty} \mu_{r_{n}, \theta_{n}, y_{n}} \text { exists if and only if } \lim _{n \rightarrow \infty} \theta_{n} \text { exists. }
$$

Thus, the Martin boundary at infinity for $\frac{1}{2} \Delta$ in $\Omega$ with the mixed Dirichlet and Neumann boundary conditions is isomorphic to $S^{d-1}$. Furthermore, every Martin boundary point is minimal, so the set of minimal elements generating the cone $\mathcal{C}_{P}\left(\Omega, \Gamma_{D}, \Gamma_{N}\right)$ is isomorphic to $S^{d-1}$.

ii. If

$$
\sum_{n=1}^{\infty} \frac{\left|\log \rho_{n}\right|^{\frac{1}{2}}}{n^{2}}=\infty
$$

then for any sequence $\left\{r_{n}, \theta_{n}, y_{n}\right\}$ satisfying $\lim _{n \rightarrow \infty} r_{n}=\infty$,

$$
\lim _{n \rightarrow \infty} \mu_{r_{n}, \theta_{n}, y_{n}} \text { exists. }
$$

Thus, the Martin boundary at infinity for $\frac{1}{2} \Delta$ in $\Omega$ with the mixed Dirichlet and Neumann boundary conditions consists of one point, and the cone $\mathcal{C}_{P}\left(\Omega, \Gamma_{D}, \Gamma_{N}\right)$ is one-dimensional.

Remark 1 . The connection between the weak convergence of exterior harmonic measures and the Martin boundary, presented before Theorem 1, does not distinguish minimal Martin boundary points from non-minimal ones, yet in part (i) of Theorem 2, it is stated that all the Martin boundary points are minimal. The reason for this is that at least one point must be minimal, of course, and then by spherical symmetry they all must in fact be minimal.

Remark 2. By Hölder's inequality with $p=\frac{3}{2}$ and $q=3$, we have

$$
\begin{aligned}
\infty & =\sum_{n=1}^{\infty} \frac{1}{n}=\sum_{n=1}^{\infty} \frac{\left|\log \rho_{n}\right|^{\frac{1}{3}}}{n^{\frac{4}{3}}} \frac{n^{\frac{1}{3}}}{\left|\log \rho_{n}\right|^{\frac{1}{3}}} \\
& \leq\left(\sum_{n=1}^{\infty} \frac{\left|\log \rho_{n}\right|^{\frac{1}{2}}}{n^{2}}\right)^{\frac{2}{3}}\left(\sum_{n=1}^{\infty} \frac{n}{\left|\log \rho_{n}\right|}\right)^{\frac{1}{3}} .
\end{aligned}
$$

From this it follows that if $\sum_{n=1}^{\infty} \frac{n}{\log \rho_{n} \mid}<\infty$, then $\sum_{n=1}^{\infty} \frac{\left|\log \rho_{n}\right|^{\frac{1}{2}}}{n^{2}}=\infty$. In light of Theorems 1 and 2, the above calculation verifies the existence of two threshold values as explained at the beginning of this section, following (1.1). To summarize, the following three regimes exist when $d \geq 3$ : 
1. (Neumann paradigm) If

$$
\sum_{n=1}^{\infty} \frac{n}{\left|\log \rho_{n}\right|}<\infty
$$

then $\mathcal{C}_{B}\left(\Omega, \Gamma_{D}, \Gamma_{N}\right)$ and $\mathcal{C}_{P}\left(\Omega, \Gamma_{D}, \Gamma_{N}\right)$ are both one-dimensional (as in the case of the Neumann boundary condition on the entire boundary). In particular, this occurs if $\rho_{n}=\exp \left(-n^{l}\right)$ with $l>2$.

2. (Dirichlet paradigm) If

$$
\sum_{n=1}^{\infty} \frac{\left|\log \rho_{n}\right|^{\frac{1}{2}}}{n^{2}}<\infty
$$

then $\mathcal{C}_{B}\left(\Omega, \Gamma_{D}, \Gamma_{N}\right)=\varnothing$ and the set of minimal elements generating $\mathcal{C}_{P}\left(\Omega, \Gamma_{D}, \Gamma_{N}\right)$ is isomorphic to $S^{d-1}$ (as in the case of the Dirichlet boundary condition on the entire boundary). In particular, this occurs if $\rho_{n}=\exp \left(-n^{l}\right)$ with $l<2$.

3. (Hybrid paradigm) If

$$
\sum_{n=1}^{\infty} \frac{n}{\left|\log \rho_{n}\right|}=\infty \quad \text { and } \quad \sum_{n=1}^{\infty} \frac{\left|\log \rho_{n}\right|^{\frac{1}{2}}}{n^{2}}=\infty,
$$

then $\mathcal{C}_{B}\left(\Omega, \Gamma_{D}, \Gamma_{N}\right)=\varnothing$ and $\mathcal{C}_{P}\left(\Omega, \Gamma_{D}, \Gamma_{N}\right)$ is one-dimensional. In particular, this occurs if $\rho_{n}=\exp \left(-n^{2}\right)$.

When $d=2$, it's easy to show that $\mathcal{C}_{B}\left(\Omega, \Gamma_{D}, \Gamma_{N}\right)=\varnothing$ as soon as there is at least one interval of Dirichlet boundary condition (see the beginning of the proof of part ii of Theorem 1). If $\sum_{n=1}^{\infty} \frac{\left|\log \rho_{n}\right|^{\frac{1}{2}}}{n^{2}}=\infty$, then $\mathcal{C}_{B}\left(\Omega, \Gamma_{D}, \Gamma_{N}\right)=\varnothing$ and $\mathcal{C}_{P}\left(\Omega, \Gamma_{D}, \Gamma_{N}\right)$ is one-dimensional (the hybrid paradigm), while if $\sum_{n=1}^{\infty} \frac{\left|\log \rho_{n}\right|^{\frac{1}{2}}}{n^{2}}<$ $\infty$, then $\mathcal{C}_{B}\left(\Omega, \Gamma_{D}, \Gamma_{N}\right)=\varnothing$ and the set of minimal elements generating $\mathcal{C}_{P}\left(\Omega, \Gamma_{D}, \Gamma_{N}\right)$ is isomorphic to $S^{d-1}$ (the Dirichlet paradigm).

We now state versions of Theorems 1 and 2 without Assumptions 1 and 2.

Theorem 1'. Let $\Gamma_{D}$ be as in (1.1) and let $\rho_{n}=b_{n}-a_{n}$.

i. If $d \geq 3$ and

$$
\sum_{n=1}^{\infty} \frac{n}{\left|\log \rho_{n}\right|}<\infty
$$

then

$$
P_{z}(\sigma<\infty)<1
$$

ii. Let $q \in(0,1)$ and define

$$
\rho_{q n, n}^{-}=\min _{k \in\{[q n], \ldots, n\}} \rho_{k} .
$$

If $d=2$, or if $d \geq 3$ and for some $q \in(0,1)$,

$$
\sum_{n=1}^{\infty} \frac{n}{\left|\log \rho_{q n, n}^{-}\right|}=\infty
$$

then

$$
P_{z}(\sigma<\infty)=1
$$



(ii).

Thus, by $(1.2), \mathcal{C}_{B}\left(\Omega, \Gamma_{D}, \Gamma_{N}\right) \neq \varnothing$ in part (i) and $\mathcal{C}_{B}\left(\Omega, \Gamma_{D}, \Gamma_{N}\right)=\varnothing$ in part

Theorem 2'. Let $\Gamma_{D}$ be as in (1.1) and let $\rho_{n}=b_{n}-a_{n}$.

i. Let $q>1$ and define

$$
\rho_{n, q n}^{-}=\min _{k \in\{n, \ldots,[q n]\}} \rho_{k} .
$$

If, for some $q>1$,

$$
\sum_{n=1}^{\infty} \frac{\left|\log \rho_{n, q n}^{-}\right|^{\frac{1}{2}}}{n^{2}} \vee \frac{\left|\log \rho_{n, q n}^{-}\right|}{n^{3}}<\infty,
$$

then for any sequence $\left\{r_{n}, \theta_{n}, y_{n}\right\}$ satisfying $\lim _{n \rightarrow \infty} r_{n}=\infty$,

$$
\lim _{n \rightarrow \infty} \mu_{r_{n}, \theta_{n}, y_{n}} \text { exists if and only if } \lim _{n \rightarrow \infty} \theta_{n} \text { exists. }
$$

The Martin boundary is as described in part (i) of Theorem 2.

ii. Let $q>1$ and define

$$
\rho_{n, q n}^{+}=\max _{k \in\{n, \ldots,[q n]\}} \rho_{k} .
$$

If, for some $q>1$,

$$
\sum_{n=1}^{\infty} \frac{\left|\log \rho_{n, q n}^{+}\right|^{\frac{1}{2}}}{n^{2}} \wedge \frac{1}{n}=\infty,
$$

then for any sequence $\left\{r_{n}, \theta_{n}, y_{n}\right\}$ satisfying $\lim _{n \rightarrow \infty} r_{n}=\infty$,

$$
\lim _{n \rightarrow \infty} \mu_{r_{n}, \theta_{n}, y_{n}} \text { exists. }
$$

The Martin boundary is as described in part (ii) of Theorem 2.

It is easy to see that under Assumption 1, the convergence/divergence specifications in Theorems $1^{\prime}$ and $2^{\prime}$ are equivalent to the corresponding ones with $\rho_{n}$ in place of $\rho_{q n, n}^{-}, \rho_{n, q n}^{-}$or $\rho_{n, q n}^{+}$, and that under Assumptions 1 and 2, the convergence/divergence specifications in Theorem $2^{\prime}$ are equivalent to the corresponding ones with $\frac{\left|\log \rho_{n, q n}^{-}\right|^{\frac{1}{2}}}{n^{2}} \vee \frac{\left|\log \rho_{n, q n}^{-}\right|}{n^{3}}$ and $\frac{\left|\log \rho_{n, q n}^{+}\right|^{\frac{1}{2}}}{n^{2}} \wedge \frac{1}{n}$ replaced by $\frac{\left|\log \rho_{n}\right|^{\frac{1}{2}}}{n^{2}}$. Thus, with the exception of the claim in part i of Theorem 1 stating that $\mathcal{C}_{B}\left(\Omega, \Gamma_{D}, \Gamma_{N}\right)$ is exactly one-dimensional, Theorems 1 and 2 follow directly from Theorems $1^{\prime}$ and $2^{\prime}$. The one-dimensionality of $\mathcal{C}_{B}\left(\Omega, \Gamma_{D}, \Gamma_{N}\right)$ follows from the second remark after Theorem 2, which indicates that if part $\mathrm{i}$ of Theorem 1 holds, then part ii of Theorem 2 must hold.

Remark. The reason we can't say that $C_{B}\left(\Omega, \Gamma_{0}, \Gamma_{N}\right)$ is exactly one-dimensional in part $\mathrm{i}$ of Theorem $1^{\prime}$ is that (1.10) does not quite imply that (1.13) holds. The reason $\rho_{n}$ does not need to be replaced in part $\mathrm{i}$ of Theorem $1^{\prime}$ is due to a correlation inequality used in its proof.

We conclude this introductory section by sketching what occurs in the two cases when the Neumann condition (reflection) or the Dirichlet condition (killing) is imposed along the entire boundary. Denote by $h_{N}$ and $h_{D}$ the $h$-function appearing 
in (1.3) for the Neumann and the Dirichlet cases respectively. In the Neumann case, we have

$$
h_{N}(r, \theta, y)=h_{N}(r)= \begin{cases}1, & \text { if } d=2, \\ r^{2-d}, & \text { if } d \geq 3\end{cases}
$$

The $h_{N}$-transformed Laplacian, $\frac{1}{2} \Delta^{h_{N}}=\frac{1}{2} \Delta+\frac{\nabla h_{N}}{h_{N}} \cdot \nabla$, is given by

$$
\frac{1}{2} \Delta^{h_{N}}=\left(\frac{1}{2} \frac{\partial^{2}}{\partial r^{2}}+\frac{3-d}{2 r} \frac{\partial}{\partial r}\right)+\frac{1}{2} \frac{\partial^{2}}{\partial y^{2}}+\frac{1}{2 r^{2}} \Delta_{S^{d-1}}
$$

In the sequel, we will need to work with the diffusion generated by $\Delta^{h_{N}}$; we will denote the probabilities and expectations associated with $\frac{1}{2} \Delta^{h_{N}}$ by $P_{.^{h_{N}}}$ and $E_{.^{h_{N}}}$.

In the Dirichlet case, we have

$$
h_{D}(r, \theta, y)=h(r, y)=g(r) \cos (\pi y / 2),
$$

where $g$ is the minimal positive solution to the equation $g^{\prime \prime}+\frac{d-1}{r} g^{\prime}-\frac{\pi^{2}}{4} g=0$ in $(1, \infty)$ with $g(1)=1$. The $h_{D}$-transformed Laplacian is given by

$$
\frac{1}{2} \Delta^{h_{D}}=\left(\frac{1}{2} \frac{\partial^{2}}{\partial r^{2}}+\frac{d-1}{2 r} \frac{\partial}{\partial r}+\frac{g^{\prime}(r)}{g(r)} \frac{\partial}{\partial r}\right)+\left(\frac{1}{2} \frac{\partial^{2}}{\partial y^{2}}-\frac{\pi}{2} \tan (\pi y / 2) \frac{\partial}{\partial y}\right)+\frac{1}{2 r^{2}} \Delta_{S^{d-1}}
$$

Using the fact that $\frac{g^{\prime}}{g}$ satisfies a Ricatti equation, it is not hard to show that there exist constants $c_{1}, c_{2}>0$ such that $c_{1} \leq-\frac{g^{\prime}}{g}(r) \leq c_{2}$, for all $r \geq 1$ [3, pp. 394-395].

Thus, the negative drift in the $r$-direction of $\Delta^{h_{N}}$ is only on the order of $\frac{1}{r}$, while that of $\Delta^{h_{D}}$ is on the order 1 . Consequently, the conditioned Brownian motion reaches $\bar{\Omega}_{0}$ much more quickly in the latter case. Denote by $\mu_{z}^{N}$ and $\mu_{z}^{D}$ the conditioned exterior harmonic measures for the Neumann boundary condition case and the Dirichlet boundary condition case respectively. It turns out that in either case, as $|z| \rightarrow \infty$, the $y$-marginal of the conditioned exterior harmonic measure approaches the ergodic equilibrium measure for the one-dimensional process in the $y$ variable; however, the $\theta$-marginal approaches the normalized uniform measure on the sphere only in the latter case. More precisely, in the Neumann case, $\lim _{r \rightarrow \infty} \mu_{r, \theta_{0}, y_{0}}^{N}(d \theta, d y)=\frac{1}{2} U(d \theta) d y$, where $U$ is the uniform probability measure on $S^{d-1}$, whereas in the Dirichlet case, $\lim _{r \rightarrow \infty} \mu_{r, \theta_{0}, y_{0}}^{D}(d \theta, d y)=$ $U_{\theta_{0}}(d \theta) \cos ^{2}(\pi y / 2) d y$, where $U_{\theta}$ is a probability distribution and $U_{\theta_{1}} \neq U_{\theta_{2}}$, for $\theta_{1} \neq \theta_{2}$. It thus follows that, in the former case, the Martin boundary at infinity is one point while in the latter case it is isomorphic to $S^{d-1}$ (see [3]).

Thus the intuition for the case under study is clear: the larger (smaller) $\Gamma_{D}$, the more rapidly (slowly) the conditioned process reaches $\bar{\Omega}_{0}$. As Theorems 2 and $2^{\prime}$ demonstrate, there is a threshold size for $\Gamma_{D}$ above which the $\theta$-component of the conditioned exterior harmonic measure will approach a limit dependent on the initial $\theta$-value, giving a Martin boundary isomorphic to $S^{d-1}$, and below which it will approach the normalized uniform measure on the sphere irrespective of the initial $\theta$-value, giving a one point Martin boundary. For a related work which uses this method to study positive harmonic functions with the Dirichlet boundary condition in a horn-shaped domain, see [1]. 
For the proofs of the two theorems, in light of condition (1.1), we may assume without loss of generality and in order to simplify notation that

$$
n+\frac{1}{4}<a_{n}<b_{n}<n+\frac{3}{4}, n \geq 1
$$

Note that the process $(r(t), y(t))$ is Markovian in its own right under $P_{r, \theta, y}$, under $P_{r, \theta, y}^{h_{N}}$, and under $P_{r, \theta, y}^{h}$ and that the process $r(t)$ is Markovian in its own right under $P_{r, \theta, y}$, and under $P_{r, \theta, y}^{h_{N}}$. Thus, in the sequel, when appropriate, we will use the notation $P_{r, y}, P_{r, y}^{h_{N}}, P_{r, y}^{h}, P_{r}$, and $P_{r}^{h_{N}}$. (In particular, $\theta$ plays no role at all in Theorem $1^{\prime}$.) The following lemma will be used for the proofs of both theorems.

Lemma 1. Let $\left\{\left(a_{k}, b_{k}\right)\right\}_{k=1}^{\infty}$ be a sequence of intervals satisfying $(1.16)$. Let $\mathcal{P}_{n, y}(\cdot)$ be a generic notation indicating any of the following probabilities:

$$
P_{n, y}(\cdot), P_{n, y}^{h_{N}}(\cdot), P_{n, y}\left(\cdot \mid \tau_{n \pm 1}<\tau_{n \mp 1}\right), P^{h_{N}}\left(\cdot \mid \tau_{n \pm 1}<\tau_{n \mp 1}\right) .
$$

Then there exist positive constants $c, C$ depending only on the dimension $d$ such that

$$
\begin{aligned}
& \frac{c}{\left|\log \rho_{n}\right|} \leq \mathcal{P}_{n, y}\left(\sigma<\tau_{n+1} \wedge \tau_{n-1}\right) \leq C\left(\frac{1}{\left|\log \rho_{n}\right|}+\frac{1}{\left|\log \rho_{n-1}\right|}\right) \\
& \text { for } y \in(-1,1), n=2,3, \ldots,
\end{aligned}
$$

where $\rho_{n}=b_{n}-a_{n}$.

We postpone the longish proof of Lemma 1 until section 4 at the end of the paper. Theorem $1^{\prime}$ is proved in section two and Theorem $2^{\prime}$ is proved in section 3 .

\section{Proof of Theorem $1^{\prime}$}

We prove each of the two parts separately.

Proof of part i. Define stopping times

$$
\begin{aligned}
& \nu_{0}=\inf \{t \geq 0: r(t) \in Z\}, \\
& \nu_{j+1}=\inf \left\{t>\nu_{j}:\left|r(t)-r\left(\nu_{j}\right)\right|=1\right\}, \text { for } j \geq 0 .
\end{aligned}
$$

For $k \geq 1$, let $U_{k}$ denote the total number of upcrossings of the interval $[k, k+1]$ by $r(t)$; that is,

$$
U_{k}=\operatorname{card}\left\{j: r\left(\nu_{j}\right)=k, r\left(\nu_{j+1}\right)=k+1\right\} .
$$

Similarly, let $D_{k}$ denote the total number of downcrossings of $[k, k+1]$; that is,

$$
D_{k}=\operatorname{card}\left\{j: r\left(\nu_{j}\right)=k+1, r\left(\nu_{j+1}\right)=k\right\} .
$$

Since $\lim _{t \rightarrow \infty} r(t)=\infty$, a.s. $P_{r, y}$ (recall that $d \geq 3$ ), it follows that

$$
U_{k}=D_{k}+1 \text { a.s. } P_{r, y}, \text { for } r \leq k \text { and } k \geq 1 \text {. }
$$

Letting $U_{0}=1$ for convenience, note also that under $P_{1, y}$, for $k=1,2, \ldots$, the number of $j$ 's for which $r\left(\nu_{j}\right)=k$ is equal to $D_{k}+U_{k-1}$. Using these facts and the 
strong Markov property gives

$$
\begin{aligned}
& P_{1, y}(\sigma=\infty)=E_{1, y} \prod_{j=0}^{\infty} P_{r\left(\nu_{j}\right), y\left(\nu_{j}\right)}\left(\nu_{j+1}<\sigma\right) \\
& \geq E_{1, y} \prod_{j=0}^{\infty} \inf _{y^{\prime}} P_{r\left(\nu_{j}\right), y^{\prime}}\left(\tau_{r\left(\nu_{j}\right)-1} \wedge \tau_{r\left(\nu_{j}\right)+1}<\sigma\right) \\
& =E_{1, y} \prod_{k=1}^{\infty}\left(\inf _{y^{\prime}} P_{k, y^{\prime}}\left(\tau_{k-1} \wedge \tau_{k+1}<\sigma\right)\right)^{D_{k}+U_{k-1}} \\
& =E_{1, y} \prod_{k=1}^{\infty}\left(\inf _{y^{\prime}} P_{k, y^{\prime}}\left(\tau_{k-1} \wedge \tau_{k+1}<\sigma\right)\right)^{U_{k-1}+U_{k}-1} .
\end{aligned}
$$

In order to continue the above calculation, we need a correlation inequality for the random variables $U_{k}$.

Lemma 2. The random variables $\left\{U_{k}\right\}_{k=0}^{\infty}$ satisfy the following correlation inequality: For any positive integers $k$ and $n$, nonnegative numbers $m_{1}, m_{2}$ and positive numbers $\left\{c_{j}\right\}_{j=0}^{n}$,

$$
P_{1, y}\left(c_{0} U_{k} \geq m_{1}, \sum_{j=1}^{n} c_{j} U_{k+j} \geq m_{2}\right) \geq P_{1, y}\left(c_{0} U_{k} \geq m_{1}\right) P_{1, y}\left(\sum_{j=1}^{n} c_{j} U_{k+j} \geq m_{2}\right) .
$$

Proof. In the proof, we will use without comment the fact that the distribution of $U_{j}$ under $P_{l, y}$ does not depend on $y \in(-1,1)$ or on $l \leq j$. Without loss of generality, assume that $\frac{m_{1}}{c_{0}}$ is a positive integer. Let $\eta_{1}$ be the time of the $\frac{m_{1}}{c_{0}}$-th upcrossing of $[k, k+1]$ if there are at least that many upcrossings, and let $\eta_{1}$ be equal to $\infty$ otherwise. Since $r\left(\eta_{1}\right)=k+1$ if $\eta_{1}<\infty$ and since up until $\eta_{1}$ there may have already been some upcrossings of intervals $[k+m, k+m+1]$, for $m \geq 1$, it follows from the strong Markov property that

$$
\begin{aligned}
& P_{1, y}\left(c_{0} U_{k} \geq m_{1}, \sum_{m=1}^{n} c_{m} U_{k+m} \geq m_{2} \geq m_{2} \mid \mathcal{F}_{\eta_{1}}\right) \\
& \geq 1_{c_{0} U_{k} \geq m_{1}} P_{k+1, y}\left(\sum_{m=1}^{n} c_{m} U_{k+m} \geq m_{2}\right) \\
& =1_{c_{0} U_{k} \geq m_{1}} P_{1, y}\left(\sum_{m=1}^{n} c_{m} U_{k+m} \geq m_{2}\right) .
\end{aligned}
$$

Taking expectations of both sides above, the result follows.

The following lemma gives the tool for applying the above correlation inequality.

Lemma 3. Let $\left\{\alpha_{j}\right\}_{j=1}^{\infty}$ and $\left\{\beta_{j}\right\}_{j=1}^{\infty}$ be increasing sequences of real numbers, and let $\mu$ and $\nu$ be probability distributions on $\left\{\alpha_{j}\right\}_{j=1}^{\infty} \times\left\{\beta_{j}\right\}_{j=1}^{\infty}$. Assume that each marginal of $\mu$ coincides with the corresponding marginal of $\nu$. Assume in addition that

$$
\sum_{l=j, r=k}^{\infty} \mu\left(\alpha_{l}, \beta_{r}\right) \geq \sum_{l=j, r=k}^{\infty} \nu\left(\alpha_{l}, \beta_{r}\right), \text { for all } j, k
$$


Let $G$ be a bounded function on $\left\{\alpha_{j}\right\}_{j=1}^{\infty} \times\left\{\beta_{j}\right\}_{j=1}^{\infty}$ whose mixed discrete partial derivative is nonnegative; that is,

$$
\begin{aligned}
G_{12}\left(\alpha_{j}, \beta_{j}\right) \equiv & G\left(\alpha_{j+1}, \beta_{j+1}\right)-G\left(\alpha_{j+1}, \beta_{j}\right) \\
& -G\left(\alpha_{j}, \beta_{j+1}\right)+G\left(\alpha_{j}, \beta_{j}\right) \geq 0, \text { for all } j .
\end{aligned}
$$

Then

$$
\sum_{j, k=1}^{\infty} G\left(\alpha_{j}, \beta_{k}\right) \mu\left(\alpha_{j}, \beta_{k}\right) \geq \sum_{j, k=1}^{\infty} G\left(\alpha_{j}, \beta_{k}\right) \nu\left(\alpha_{j}, \beta_{k}\right) .
$$

Proof. Let $\eta$ denote either $\mu$ or $\nu$ in the calculation that follows. Let $\eta_{i}$ denote the $i$-th marginal of $\eta$ and let $\hat{\eta}_{2}\left(\beta_{k}\right)=\sum_{l \geq k} \eta_{2}\left(\beta_{l}\right)$ By the assumption on the marginals of $\mu$ and $\nu$, it follows that $\eta_{1}$ and $\hat{\eta}_{2}$ are the same for $\mu$ and $\nu$. A discrete integration by parts formula yields

$$
\begin{aligned}
& \sum_{j, k=1}^{\infty} G\left(\alpha_{j}, \beta_{k}\right) \eta\left(\alpha_{j}, \beta_{k}\right)=\sum_{j=1}^{\infty} G\left(\alpha_{j}, \beta_{1}\right) \eta_{1}\left(\alpha_{j}\right) \\
& +\sum_{k=2}^{\infty}\left(G\left(\alpha_{1}, \beta_{k}\right)-G\left(\alpha_{1}, \beta_{k-1}\right)\right) \hat{\eta}_{2}\left(\beta_{k}\right)+\sum_{j, k=2}^{\infty} G_{12}\left(\alpha_{j}, \beta_{k}\right)\left(\sum_{l=j, r=k}^{\infty} \eta\left(\alpha_{l}, \beta_{r}\right)\right) .
\end{aligned}
$$

The lemma now follows from (2.3), (2.4), and (2.5).

We now apply Lemmas 2 and 3 to (2.1).

Lemma 4. Let $\gamma_{k}=\sup _{y} P_{k, y}\left(\sigma<\tau_{k-1} \wedge \tau_{k+1}\right)$. Then

$$
P_{1, y}(\sigma=\infty) \geq\left(1-\gamma_{1}\right)^{\frac{1}{2}} \prod_{k=1}^{\infty} E_{1, y} \exp \left(\left(U_{k}-\frac{1}{2}\right) \log \left(1-\gamma_{k}\right)\left(1-\gamma_{k+1}\right)\right) .
$$

Proof. Let $\delta_{k}=1-\gamma_{k}=\inf _{y} P_{k, y}\left(\tau_{k-1} \wedge \tau_{k+1}<\sigma\right)$. By (2.1), we have

$$
P_{1, y}(\sigma=\infty) \geq \lim _{N \rightarrow \infty} E_{1, y} \prod_{k=1}^{N} \delta_{k}^{U_{k-1}+U_{k}-1} .
$$

We write

$$
\begin{aligned}
& E_{1, y} \prod_{k=1}^{N} \delta_{k}^{U_{k-1}+U_{k}-1} \\
& =\delta_{1}^{\frac{1}{2}} E_{1, y} \exp \left(\sum_{k=1}^{N-1}\left(\log \delta_{k} \delta_{k+1}\right)\left(U_{k}-\frac{1}{2}\right)\right)+\left(\log \delta_{N}\right)\left(U_{N}-\frac{1}{2}\right) .
\end{aligned}
$$

We now apply Lemmas 2 and 3. Let $G(\alpha, \beta)=\exp (-\alpha-\beta)$ and note that $G$ satisfies (2.4). Let $\mu$ be the two-dimension distribution induced by $-\left(\log \delta_{1} \delta_{2}\right) U_{1}$ and $-\sum_{k=2}^{N-1}\left(\log \delta_{k} \delta_{k+1}\right) U_{k}-\left(\log \delta_{N}\right) U_{N}$ under $P_{1, y}$, and let $\nu$ be the twodimensional product distribution induced by $-\left(\log \delta_{1} \delta_{2}\right) U_{1}$ under $P_{1, y}$ and $-\sum_{k=2}^{N-1}\left(\log \delta_{k} \delta_{k+1}\right) U_{k}-\left(\log \delta_{N}\right) U_{N}$ under $P_{1, y}$. Note that $\mu$ and $\nu$ are supported on a set of the form $\left\{\alpha_{j}\right\}_{j=1}^{\infty} \times\left\{\beta_{j}\right\}_{j=1}^{\infty}$ as specified in Lemma 3 . In light of the 
correlation inequality (2.2), it follows that (2.3) holds for the above choice of $\mu$ and $\nu$. Thus, applying the conclusion of Lemma 3 gives

$$
\begin{aligned}
& E_{1, y} \exp \left(\left(\log \delta_{1} \delta_{2}\right) U_{1}\right) \exp \left(\left(\log \delta_{N}\right) U_{N}+\sum_{k=2}^{N-1}\left(\log \delta_{k} \delta_{k+1}\right) U_{k}\right) \\
& \geq E_{1, y} \exp \left(\left(\log \delta_{1} \delta_{2}\right) U_{1}\right) E_{1, y} \exp \left(\left(\log \delta_{N}\right) U_{N}+\sum_{k=2}^{N-1}\left(\log \delta_{k} \delta_{k+1}\right) U_{k}\right)
\end{aligned}
$$

The above argument using Lemma 2 and Lemma 3 can now be applied to $E_{1, y} \exp \left(\left(\log \delta_{N}\right) U_{N}+\sum_{k=2}^{N-1}\left(\log \delta_{k} \delta_{k+1}\right) U_{k}\right)$. After $N-2$ additional applications of the argument, we end up with the inequality

$$
\begin{aligned}
& E_{1, y} \exp \left(\left(\log \delta_{1} \delta_{2}\right) U_{1}\right) \exp \left(\left(\log \delta_{N}\right) U_{N}+\sum_{k=2}^{N-1}\left(\log \delta_{k} \delta_{k+1}\right) U_{k}\right) \\
& \geq E_{1, y} \exp \left(\left(\log \delta_{N}\right) U_{N}\right) \prod_{k=1}^{N-1} E_{1, y} \exp \left(\left(\log \delta_{k} \delta_{k+1}\right) U_{k}\right) .
\end{aligned}
$$

Using (2.8) in (2.7) and substituting in (2.6) proves the lemma.

The next lemma gives the distribution of $U_{k}$ under $P_{1, y}$.

Lemma 5. $P_{1, y}\left(U_{k}=n\right)=l_{k}^{n-1}\left(1-l_{k}\right)$, for $k=1,2, \ldots$ and $n=1,2, \ldots$, where $l_{k}=\left(\frac{k}{k+1}\right)^{d-2}$.

Proof. The proof follows immediately from the strong Markov property and the fact that $P_{k+1, y}\left(\tau_{k}<\infty\right)=l_{k}$.

Using Lemma 5, we calculate directly

$$
E_{1, y} \exp \left(\left(U_{k}-\frac{1}{2}\right) \log \left(1-\gamma_{k}\right)\left(1-\gamma_{k+1}\right)\right)=\frac{\left(1-\gamma_{k}\right)^{\frac{1}{2}}\left(1-\gamma_{k+1}\right)^{\frac{1}{2}}\left(1-l_{k}\right)}{1-\left(1-\gamma_{k}\right)\left(1-\gamma_{k+1}\right) l_{k}}
$$

Let $\hat{\gamma}_{k}=\max \left(\gamma_{k}, \gamma_{k+1}\right)$. Then

$$
\begin{aligned}
& \frac{\left(1-\gamma_{k}\right)^{\frac{1}{2}}\left(1-\gamma_{k+1}\right)^{\frac{1}{2}}\left(1-l_{k}\right)}{1-\left(1-\gamma_{k}\right)\left(1-\gamma_{k+1}\right) l_{k}} \geq \frac{\left(1-\hat{\gamma}_{k}\right)\left(1-l_{k}\right)}{1-\left(1-\hat{\gamma}_{k}\right)^{2} l_{k}} \\
& =\frac{1-l_{k}-\hat{\gamma}_{k}+l_{k} \hat{\gamma}_{k}}{1-l_{k}-l_{k} \hat{\gamma}_{k}^{2}+2 l_{k} \hat{\gamma}_{k}}=1-\frac{\hat{\gamma}_{k}-l_{k} \hat{\gamma}_{k}^{2}+l_{k} \hat{\gamma}_{k}}{1-l_{k}-l_{k} \hat{\gamma}_{k}^{2}+2 l_{k} \hat{\gamma}_{k}},
\end{aligned}
$$

and, since $\hat{\gamma}_{k}, l_{k} \leq 1$,

$$
\frac{\hat{\gamma}_{k}-l_{k} \hat{\gamma}_{k}^{2}+l_{k} \hat{\gamma}_{k}}{1-l_{k}-l_{k} \hat{\gamma}_{k}^{2}+2 l_{k} \hat{\gamma}_{k}} \leq \frac{2 \hat{\gamma}_{k}}{1-l_{k}}
$$

Using (2.9)-(2.11) in Lemma 4, we obtain

$$
P_{1, y}(\sigma=\infty) \geq\left(1-\gamma_{1}\right)^{\frac{1}{2}} \prod_{k=1}^{\infty}\left(1-\frac{2 \hat{\gamma}_{k}}{1-l_{k}}\right)
$$

Recalling that $\hat{\gamma}_{k}=\max \left(\gamma_{k}, \gamma_{k+1}\right)$, recalling the definition of $\gamma_{k}$ in Lemma 4, and using Lemma 1, we have

$$
\hat{\gamma}_{k} \leq 2 C\left(\frac{1}{\left|\log \rho_{k-1}\right|}+\frac{1}{\left|\log \rho_{k}\right|}+\frac{1}{\left|\log \rho_{k+1}\right|}\right) .
$$


Also, there exists a $c>0$ such that

$$
1-l_{k}=1-\left(\frac{k}{k+1}\right)^{d-2} \geq \frac{c}{k} \text {. }
$$

From (2.12)-(2.14) and assumption (1.10) in part i of the theorem, it follows that $P_{1, y}(\sigma=\infty)>0$. This proves part i.

Proof of part ii. If $d=2$, then the radial process is recurrent and a simple application of the strong Markov property shows that $P_{z}(\sigma<\infty)=1$ as soon as there is at least one interval of Dirichlet boundary condition. We leave this to the reader. Thus, from now on, we assume that $d \geq 3$.

In order to keep the notation simpler, we will assume that (1.11) holds for $q=\frac{1}{2}$. It will be clear how to amend the proof for other values of $q$. By the strong Markov property and the transience of the radial process, we have

$$
P_{2, y}(\sigma=\infty) \leq \prod_{n=2}^{\infty} \sup _{y^{\prime}} P_{n, y^{\prime}}\left(\tau_{n+1}<\sigma\right) \leq \prod_{n=2}^{\infty} \sup _{y^{\prime}} P_{n, y^{\prime}}\left(\tau_{n+1} \wedge \tau_{\left[\frac{n}{2}\right]}<\sigma\right) .
$$

Recall the definition of $\left\{\nu_{n}\right\}_{n=0}^{\infty}$ at the beginning of the proof of part i. Define $V_{n}, n=2,3, \ldots$, by

$$
V_{n}=j, \text { if } \nu_{j}=\tau_{n+1} \wedge \tau_{\left[\frac{n}{2}\right]} .
$$

Thus, under $P_{n, y^{\prime}}, V_{n}$ counts the number of times the process $r(t)$ upcrosses or downcrosses an interval of the form $[j, j+1]$ before reaching either $n+1$ or $\left[\frac{n}{2}\right]$. We write

$$
P_{n, y^{\prime}}\left(\tau_{n+1} \wedge \tau_{\left[\frac{n}{2}\right]}<\sigma\right)=\sum_{m=1}^{\infty} P_{n, y^{\prime}}\left(\tau_{n+1} \wedge \tau_{\left[\frac{n}{2}\right]}<\sigma, V_{n}=m\right) .
$$

Now for any sequence $\left\{r_{j}\right\}_{j=0}^{m}$ satisfying $r_{0}=n, r_{m}=n+1$ or $\left[\frac{n}{2}\right], r_{j} \in$ $\left\{\left[\frac{n}{2}\right]+1, \ldots, n\right\}$, for $j \in\{1,2, \ldots, m-1\}$, and $\left|r_{j}-r_{j-1}\right|=1$, we have

$$
\begin{aligned}
& P_{n, y^{\prime}}\left(\tau_{n+1} \wedge \tau_{\left[\frac{n}{2}\right]}<\sigma, V_{n}=m \mid V_{n}=m,\left\{r\left(\nu_{j}\right)\right\}_{j=0}^{m-1}=\left\{r_{j}\right\}_{j=0}^{m-1}\right) \\
& =E_{n, y^{\prime}} \prod_{j=0}^{m-1} P_{r_{j}, y\left(\nu_{j}\right)}\left(\tau_{r_{j}-1} \wedge \tau_{r_{j}+1}<\sigma \mid \tau_{r_{j}-1} \wedge \tau_{r_{j}+1}=\tau_{r_{j+1}}\right) .
\end{aligned}
$$

An application of the strong Markov property gives

$$
\begin{aligned}
& P_{r_{j}, y}\left(\tau_{r_{j}-1} \wedge \tau_{r_{j}+1}<\sigma \mid \tau_{r_{j}-1} \wedge \tau_{r_{j}+1}=\tau_{r_{j+1}}\right) \\
& =1-P_{r_{j}, y}\left(\sigma<\tau_{r_{j}-1} \wedge \tau_{r_{j}+1} \mid \tau_{r_{j}-1} \wedge \tau_{r_{j}+1}=\tau_{r_{j+1}}\right) \\
& =1-\frac{E_{r_{j}, y}\left(P_{r(\sigma), y(\sigma)}\left(\tau_{r_{j}-1} \wedge \tau_{r_{j}+1}=\tau_{r_{j+1}}\right) ; \sigma<\tau_{r_{j}-1} \wedge \tau_{r_{j}+1}\right)}{P_{r_{j}, y}\left(\tau_{r_{j}-1} \wedge \tau_{r_{j}+1}=\tau_{r_{j+1}}\right)} .
\end{aligned}
$$

Define

$$
h_{k}(r)=P_{r, y}\left(\tau_{k-1}<\tau_{k+1}\right), \text { for } r \in(k-1, k+1) .
$$

(The right-hand side above is independent of $y$.) Then $h_{k}$ satisfies

$$
\begin{aligned}
& \frac{1}{2} h_{k}^{\prime \prime}+\frac{d-1}{2 r} h_{k}^{\prime}=0, \quad r \in(k-1, k+1), \\
& h(k-1)=1, \quad h(k+1)=0 .
\end{aligned}
$$


Note that $P_{r(\sigma), y(\sigma)}\left(\tau_{r_{j}-1} \wedge \tau_{r_{j}+1}=\tau_{r_{j+1}}\right)$ is either equal to $h_{r_{j}}(r(\sigma))$ or to $1-$ $h_{r_{j}}(r(\sigma))$. A direct calculation (or alternatively, standard elliptic theory) shows that

$$
0<\inf _{k \geq 2} \inf _{r \in\left(k-\frac{3}{4}, k+\frac{3}{4}\right)} h_{k}(r)<\sup _{k \geq 2} \sup _{r \in\left(k-\frac{3}{4}, k+\frac{3}{4}\right)} h_{k}(r)<1 .
$$

In light of (1.16), $r(\sigma) \in\left(r_{j}+\frac{1}{4}, r_{j}+\frac{3}{4}\right) \cup\left(r_{j}-\frac{3}{4}, r_{j}-\frac{1}{4}\right)$ a.s. $P_{r_{j}, y_{j}}$ Thus, from (2.19)-(2.21), it follows that there exists a $c>0$, independent of $r_{j}$ and $y$, such that

$$
\begin{aligned}
& P_{r_{j}, y}\left(\tau_{r_{j}-1} \wedge \tau_{r_{j}+1}<\sigma \mid \tau_{r_{j}-1} \wedge \tau_{r_{j}+1}=\tau_{r_{j+1}}\right) \\
& \leq 1-c_{\left.k \in\left\{\frac{n}{2}\right]+1, \ldots, n\right\}, y^{\prime}} P_{k, y^{\prime}}\left(\sigma<\tau_{k-1} \wedge \tau_{k+1}\right) .
\end{aligned}
$$

Using Lemma 1 and (2.22), and recalling the definition of $\rho_{q n, n}^{-}$in the statement of part ii of the theorem, it follows that there exists a $C>0$, independent of $r_{j}$ and $y$, such that

$$
\begin{aligned}
& P_{r_{j}, y}\left(\tau_{r_{j}-1} \wedge \tau_{r_{j}+1}<\sigma \mid \tau_{r_{j}-1} \wedge \tau_{r_{j}+1}=\tau_{r_{j+1}}\right) \\
& \quad \leq 1-C\left(\max _{k \in\left\{\left[\frac{n}{2}\right], \ldots, n\right\}}\left|\log \rho_{k}\right|\right)^{-1} \\
& \quad=1-\frac{C}{\left|\log \rho_{\frac{1}{2} n, n}^{-}\right|} .
\end{aligned}
$$

From (2.17), (2.18), and (2.23), we conclude that

$$
P_{n, y^{\prime}}\left(\tau_{n+1} \wedge \tau_{\left[\frac{n}{2}\right]}<\sigma\right) \leq E_{n, y^{\prime}}\left(1-\frac{C}{\left|\log \rho_{\frac{1}{2} n, n}^{-}\right|}\right)^{V_{n}} .
$$

Using (2.15) and (2.24), along with the fact that the distribution of $V_{n}$ under $P_{n, y^{\prime}}$ does not depend on $y^{\prime}$, and along with the inequality $(1-x)^{a} \leq \exp (-a x)$, for $a>0$ and $x \in(0,1)$, we obtain

$$
P_{2, y}(\sigma=\infty) \leq \prod_{n=2}^{\infty} E_{n} \exp \left(-C \frac{V_{n}}{\left|\log \rho_{\frac{1}{2} n, n}^{-}\right|}\right) .
$$

Let $\left\{\mathcal{V}_{n}\right\}_{n=2}^{\infty}$ be a sequence of independent random variables on a probability space with measure $\mathcal{P}$ and corresponding expectation $\mathcal{E}$, and such that $\mathcal{V}_{n}$ has the distribution under $\mathcal{P}$ that $V_{n}$ has under $P_{n}$. Then (2.25) may be rewritten as

$$
P_{2, y}(\sigma=\infty) \leq \mathcal{E} \exp \left(-C \sum_{k=2}^{\infty} \frac{\mathcal{V}_{n}}{\left|\log \rho_{\frac{1}{2} n, n}^{-}\right|}\right) .
$$

To complete the proof of part ii, we will show that

$$
\sum_{k=2}^{\infty} \frac{\mathcal{V}_{n}}{\left|\log \rho_{\frac{1}{2} n, n}^{-}\right|}=\infty \text { a.s. } \mathcal{P} \text {. }
$$

We will use the following result.

Proposition 1. Let $\left\{X_{n}\right\}_{n=1}^{\infty}$ be a sequence of bounded, nonnegative, independent random variables. Then $\sum_{n=1}^{\infty} X_{n}=\infty$ a.s. if and only if $\sum_{n=1}^{\infty} E X_{n}=\infty$. 
Proof. The proof of this result must appear in the literature, but since this author found no obvious source, here is a proof. One direction is trivial. For the other direction, assume that

$$
\sum_{n=1}^{\infty} E X_{n}=\infty
$$

To show that $\sum_{n=1}^{\infty} X_{n}=\infty$ a.s., will will show that $E \exp \left(-\sum_{n=1}^{\infty} X_{n}\right)=0$. Let $M$ be an upper bound on the random variables $\left\{X_{n}\right\}_{n=1}^{\infty}$. Then by the mean value theorem, $\exp \left(-X_{n}\right) \leq\left(1-X_{n} \exp (-M)\right)$. Thus,

$$
E \exp \left(-\sum_{n=1}^{\infty} X_{n}\right)=\prod_{n=1}^{\infty} E \exp \left(-X_{n}\right) \leq \prod_{n=1}^{\infty}\left(1-\exp (-M) E X_{n}\right)=0
$$

where the last inequality follows from (2.28).

To apply Proposition 1 and prove part ii, we need one more lemma.

Lemma 6. There exists a $c>0$ such that

$$
P\left(\mathcal{V}_{n} \geq n^{2}\right) \geq \frac{c}{n}
$$

In particular then,

$$
\mathcal{E}\left(\mathcal{V}_{n} \wedge n^{2}\right) \geq c n .
$$

Proof. Since they have the same distribution, we may work with $V_{n}$ under $P_{n}$ rather than with $\mathcal{V}_{n}$ under $\mathcal{P}$. Note that $P_{n}\left(\tau_{\left[\frac{3 n}{4}\right]}<\tau_{n+1}\right)=\frac{n^{2-d}-(n+1)^{2-d}}{\left[\frac{3 n}{4}\right]^{2-d}-(n+1)^{2-d}} \geq \frac{c}{n}$, for some $c>0$. Recall from (2.16) that under $P_{\frac{3 n}{4}, y}, V_{n}$ counts the number of times the process $r(t)$ upcrosses or downcrosses an interval of the form $[j, j+1]$ before reaching either $n+1$ or $\left[\frac{n}{2}\right]$. Thus, to complete the proof of (2.29), it suffices to show that there exists a $c>0$ such that

$$
P_{\frac{3 n}{4}}\left(V_{n} \geq n^{2}\right) \geq c .
$$

But (2.31) follows as a consequence of Brownian scaling.

We can now prove (2.27) to complete the proof of part ii. By (2.29) and the Borel-Cantelli Lemma,

$$
\mathcal{P}\left(\mathcal{V}_{n} \geq n^{2} \text { i.o. }\right)=1 \text {. }
$$

Thus, (2.27) certainly holds if $\frac{n^{2}}{\left|\log \rho_{\frac{1}{2} n, n}^{-}\right|}$is unbounded. From here on, assume that $\frac{n^{2}}{\left|\log \rho_{\frac{1}{2} n, n}^{-}\right|}$is bounded. Let $X_{n} \equiv \frac{\mathcal{V}_{n} \wedge n^{2}}{\left|\log \rho_{\frac{1}{2} n, n}^{-}\right|}$. Then the sequence $X_{n}$ is bounded and by (2.30), $\mathcal{E} X_{n} \geq \frac{c n}{\left|\log \rho_{\frac{1}{2} n, n}^{-}\right|}$. By assumption (1.11) in part ii of the theorem, it follows that $\sum_{n=2}^{\infty} \mathcal{E} X_{n}=\infty$, and therefore, by Proposition 1, (2.27) holds.

\section{Proof of Theorem $2^{\prime}$}

In light of (1.4), we may use the skew product form to represent the $\theta$-component of $z(t)=(r(t), \theta(t), y(t))$ under $P_{r, \theta, y}^{h}$ as $\theta(t)=\phi\left(\int_{0}^{t} \frac{1}{r^{2}(s)} d s\right)$, where $\phi(\cdot)$ under $P_{r, \theta, y}^{h}$ is a standard Brownian motion on $S^{d-1}$, starting from $\theta \in S^{d-1}$, and independent of the Markov process $(r(\cdot), y(\cdot))$. Most of the work in the proof will 
be to show that if (1.12) holds, then $\int_{0}^{\tau_{1}} \frac{1}{r^{2}(s)} d s$ under $P_{r, y}^{h}$ is uniformly tight over $y \in(-1,1)$ as $r \rightarrow \infty$; that is,

$$
\lim _{x \rightarrow \infty} \liminf _{r \rightarrow \infty} \inf _{y} P_{r, y}^{h}\left(\int_{0}^{\tau_{1}} \frac{1}{r^{2}(s)} d s \leq x\right)=1 ;
$$

and that if (1.13) holds, then $\int_{0}^{\tau_{1}} \frac{1}{r^{2}(s)} d s$ under $P_{r, y}^{h}$ converges weakly to the $\delta$-mass at $\infty$ as $r \rightarrow \infty$, and that this convergence is uniform over $y \in(-1,1)$; that is,

$$
\lim _{r \rightarrow \infty} \inf _{y} P_{r, y}^{h}\left(\int_{0}^{\tau_{1}} \frac{1}{r^{2}(s)} d s>x\right)=1, \text { for all } x>0 .
$$

We will now complete the proof of the theorem relatively quickly using (3.1) and (3.2), and then return to the main job of proving these two results. Recall the definition of $\mu_{z}$ in (1.5). Let $\|\cdot\|$ denote a metric compatible with weak convergence on the space of probability measures on $S^{d-1} \times(-1,1)$. By standard theory, the measures $\mu_{z}$ are weakly continuous in $z$. For any fixed $R$, if we start two independent $P_{.,}^{h}$-diffusions, $(r(t), y(t))$, one from $\left(r, y_{1}\right)$ and the other from $\left(r, y_{2}\right)$, then the probability that their paths intersect before their $r$-coordinates reach $R$ converges to one as $r \rightarrow \infty$, uniformly over $y$. Indeed this follows from the topology of $R^{2}$ and the fact that a $P_{r, y}^{h}$-diffusion will hit $y=-1$ before time $\tau_{R}$ with a probability that tends to 1 as $r \rightarrow \infty$, uniformly over $y$. This latter fact, intuitively clear, and simple to prove, is left to the reader. Define

$$
\nu_{r, \theta, y}^{R}\left(d \theta^{\prime}, d y^{\prime}\right)=P_{r, \theta, y}^{h}\left(\theta\left(\tau_{R}\right) \in d \theta^{\prime}, y\left(\tau_{R}\right) \in d y^{\prime}\right) \text {, for } r>R .
$$

By the strong Markov property,

$$
\mu_{r, \theta, y}=\int \mu_{R, \theta^{\prime}, y^{\prime}} \nu_{r, \theta, y}^{R}\left(d \theta^{\prime}, d y^{\prime}\right) .
$$

Assume now first that (3.1) holds. In order to prove the theorem, we must show that, for any sequence $\left\{r_{n}, \theta_{n}, y_{n}\right\}$ satisfying $\lim _{n \rightarrow \infty} r_{n}=\infty$,

$$
\lim _{n \rightarrow \infty} \mu_{r_{n}, \theta_{n}, y_{n}} \text { exists if and only if } \lim _{n \rightarrow \infty} \theta_{n} \text { exists. }
$$

Fix $\epsilon>0$. Making a direct argument using (3.1), the strong Markov property, and a little analysis, or alternatively, chasing through the proof of (3.1) with $r=1$ replaced by $r=R$, it follows that we can choose $R_{\epsilon}$ and $r_{\epsilon}>R_{\epsilon}$ so large that

$$
\inf _{y} P_{r, y}^{h}\left(\int_{0}^{\tau_{R_{\epsilon}}} \frac{1}{r^{2}(s)} d s<\epsilon\right)>1-\epsilon, \text { for all } r \geq r_{\epsilon} .
$$

If necessary, increase $r_{\epsilon}$ so that for any $r \geq r_{\epsilon}$, the probability that two independent diffusion paths, as described above, will intersect before their $r$-coordinates reach $R_{\epsilon}$ is at least $1-\epsilon$. It then follows that, for $r \geq r_{\epsilon}$, the $\theta$-marginals of $\nu_{r, \theta, y_{1}}^{R^{\epsilon}}$ and $\nu_{r, \theta, y_{2}}^{R^{\epsilon}}$ are close to the $\delta$-measure at $\theta$, and, via coupling, that their $y$-marginals are close to one another; thus, the entire measures are close to one another. This closeness is uniform over $\theta, y_{1}$ and $y_{2}$. We conclude from the above analysis that

$$
\lim _{r \rightarrow \infty} \sup _{\theta, y_{1}, y_{2}}\left\|\mu_{r, \theta, y_{1}}-\mu_{r, \theta, y_{2}}\right\|=0 .
$$

Similarly, if $r_{2}>r_{1}>1$, then by the strong Markov property

$$
\mu_{r_{2}, \theta, y}=\int \mu_{r_{1}, \theta^{\prime}, y^{\prime}} \nu_{r_{2}, \theta, y}^{r_{1}}\left(d \theta^{\prime}, d y^{\prime}\right)
$$


Thus,

$$
\begin{aligned}
& \left\|\mu_{r_{2}, \theta_{2}, y_{2}}-\mu_{r_{1}, \theta_{1}, y_{1}}\right\|=\left\|\int\left(\mu_{r_{1}, \theta^{\prime}, y^{\prime}}-\mu_{r_{1}, \theta_{1}, y_{1}}\right) \nu_{r_{2}, \theta_{2}, y_{2}}^{r_{1}}\left(d \theta^{\prime}, d y^{\prime}\right)\right\| \\
& \leq \int\left\|\mu_{r_{1}, \theta^{\prime}, y^{\prime}}-\mu_{r_{1}, \theta_{1}, y_{1}}\right\| \nu_{r_{2}, \theta_{2}, y_{2}}^{r_{1}}\left(d \theta^{\prime}, d y^{\prime}\right) .
\end{aligned}
$$

From the fact that $(r(t), y(t))$ is a Markov process without the $\theta$-component, from the representation $\theta(t)=\phi\left(\int_{0}^{t} \frac{1}{r^{2}(s)} d s\right)$, and from (3.1), it follows that

$$
\lim _{\theta^{\prime} \rightarrow \theta_{1}} \sup _{r_{1}, \theta_{1}, y^{\prime}}\left\|\mu_{r_{1}, \theta^{\prime}, y^{\prime}}-\mu_{r_{1}, \theta_{1}, y^{\prime}}\right\|=0 .
$$

Writing $\left\|\mu_{r_{1}, \theta^{\prime}, y^{\prime}}-\mu_{r_{1}, \theta_{1}, y_{1}}\right\| \leq\left\|\mu_{r_{1}, \theta^{\prime}, y^{\prime}}-\mu_{r_{1}, \theta_{1}, y^{\prime}}\right\|+\left\|\mu_{r_{1}, \theta_{1}, y^{\prime}}-\mu_{r_{1}, \theta_{1}, y_{1}}\right\|$, noting from (3.3) that, uniformly in $\theta_{2}$ and $y_{2}$, the $\theta$-marginal of $\nu_{r_{2}, \theta_{2}, y_{2}}^{r_{1}}$ approaches the $\delta$-measure at $\theta_{2}$ as $r_{1}, r_{2} \rightarrow \infty$, and using (3.4), (3.5), and (3.6), it follows that

$$
\lim _{\substack{r_{1}, r_{2} \rightarrow \infty \\ \theta_{2} \rightarrow \theta_{1}}} \sup _{y_{1}, y_{2}, \theta_{1}}\left\|\mu_{r_{2}, \theta_{2}, y_{2}}-\mu_{r_{1}, \theta_{1}, y_{1}}\right\|=0 .
$$

Since the space $S^{d-1} \times[-1,1]$ is compact, the measures $\left\{\mu_{r, \theta, y}\right\}$ are tight. Thus, it follows from (3.7) that $\lim \mu_{r_{n}, \theta_{n}, y_{n}}$ exists whenever $r_{n} \rightarrow \infty$ and $\theta_{n}$ converges.

To complete the proof in the case that (3.1) holds, it only remains to show that $\lim _{r \rightarrow \infty} \mu_{r, \theta_{1}, y} \neq \lim _{r \rightarrow \infty} \mu_{r, \theta_{2}, y}$. To see that this is true, note from the skew product representation that the $\theta$-marginal of $\lim _{r \rightarrow \infty} \mu_{r, \theta_{i}, y}$ has a distribution of the form $\int_{0}^{\infty} \Phi(s) \eta_{i}(d s)$, where, for each $s, \Phi(s)$ is a Gaussian distribution on $S^{d-1}$, centered at $\theta_{i}$, and $\eta_{i}$ is a probability distribution on $[0, \infty)$. It follows then that the density of $\lim _{r \rightarrow \infty} \mu_{r, \theta_{i}, y}$ attains its unique maximum at $\theta_{i}$. Thus the distributions are distinct for $\theta_{1} \neq \theta_{2}$. This completes the proof in the case that (3.1) holds.

Now assume that (3.2) holds. Recall that, to prove the theorem, we must show that

$$
\lim _{n \rightarrow \infty} \mu_{r_{n}, \theta_{n}, y_{n}} \text { exists, }
$$

for any sequence $\left\{r_{n}, \theta_{n}, y_{n}\right\}$ satisfying $\lim _{n \rightarrow \infty} r_{n}=\infty$. Since the measures $\left\{\mu_{r, \theta, y}\right\}$ are tight, it is enough to show that there is only one possible limiting distribution. By the coupling argument noted above, it follows that there can only be one possible limiting $y$-marginal, and by (3.2), the skew product representation for $\theta(t)$, and the fact that $\phi(t)$ is ergodic on $S^{d-1}$, it follows that the uniform distribution is the only possible limiting $\theta$-marginal. Thus, to complete the proof, it is enough to show that any limiting distribution on $S^{d-1} \times[-1,1]$ must be a product distribution. Specifically, we will show that if

$$
\mu(d \theta, d y)=\lim _{n \rightarrow \infty} \mu_{r_{n}, \theta_{n}, y_{n}}(d \theta, d y) \equiv \lim _{n \rightarrow \infty} P_{r_{n}, \theta_{n}, y_{n}}^{h}\left(\theta\left(\tau_{1}\right) \in d \theta, y\left(\tau_{1}\right) \in d y\right)
$$

is some limiting distribution, then, for any subinterval $(a, b) \subset[-1,1]$, the conditional distribution

$$
\mu(d \theta \mid y \in(a, b)) \equiv \frac{\int_{a}^{b} \mu(d \theta, d y)}{\int_{S^{d-1}} \int_{a}^{b} \mu(d \theta, d y)}
$$

is equal to the uniform distribution. Now $\mu(d \theta \mid y \in(a, b))$ can be obtained as follows. Recall the definition of $h$ in (1.3). Define $\hat{h}(z)=P_{z}\left(\tau_{1}<\sigma, y\left(\tau_{1}\right) \in(a, b)\right)$. Whereas $P^{h}$ corresponded to the original $P$.-diffusion conditioned to hit $\{r=1\}$ before hitting $\Gamma_{D}, P^{\hat{h}}$ corresponds to the original $P$.-diffusion conditioned to hit 
$\{r=1\} \cap\{y \in(a, b)\}$ before hitting $(\{r=1\} \cap\{y \notin(a, b)\}) \cup \Gamma_{D}$. It then follows that the limit on the right-hand side of (3.8) continues to exist when $h$ is replaced by $\hat{h}$. Defining $\hat{\mu}(d \theta, d y)$ by the right-hand side of (3.8) with $h$ replaced by $\hat{h}$, it follows that $\mu(d \theta \mid y \in(a, b))$ is equal to the $\theta$-marginal of $\hat{\mu}(d \theta, d y)$.

Now the exact same argument that we give below to prove (3.2) can be used to prove that (3.2) also holds when $h$ is replaced by $\hat{h}$. Thus, just as we concluded above that the $\theta$-marginal of $\mu$ was uniform, we can conclude that the $\theta$-marginal of $\hat{\mu}$ is also uniform. This completes the proof in the case that (3.2) holds.

Proof of (3.1) under the assumption that (1.12) holds. By Chebyshev's inequality, it is enough to show that

$$
\limsup _{r \rightarrow \infty} \sup _{y} E_{r, y}^{h} \int_{0}^{\tau_{1}} \frac{1}{r^{2}(s)} d s<\infty .
$$

Define

$$
u_{n}(r, y)=E_{r, y}^{h} \int_{0}^{\tau_{1}} I_{[n-1, n]}(r(s)) d s .
$$

From the strong Markov property, it follows that (3.9) will hold if we show that

$$
\sum_{n=2}^{\infty} \frac{\sup _{y} u_{n}(n, y)}{n^{2}}<\infty .
$$

We now make a construction which will allow us to rewrite $u_{n}$ above in terms of $E_{r, y}^{h_{N}}$ instead of $E_{r, y}^{h}$. This will make things much simpler because under $E_{r, y}^{h_{N}}$ the components $r(t)$ and $y(t)$ are independent. Note that $u_{n}$ is the minimal positive solution to $\Delta^{h} u_{n}=-I_{[n-1, n]}$ with the Neumann boundary condition on $\Gamma_{N}$ and with the Dirichlet boundary condition on $\{r=1\}$. (No boundary data is given explicitly on $\Gamma_{D}$, but rather implicitly through the minimality requirement.) Recall the definition of $h_{N}$ and $\frac{1}{2} \Delta^{h_{N}}$ in (1.14) and (1.15). The measure $P^{h_{N}}$ corresponds to the original reflected diffusion conditioned on $\tau_{1}<\infty$. Define $g=\frac{h}{h_{N}}$, and note that $g$ vanishes on $\Gamma_{D}$. (In fact, $g(r, y)=P_{r, y}^{h_{N}}\left(\tau_{1}<\sigma\right)$.) Define $v_{n}=g u_{n}$. Since $\Delta^{h} u_{n}=\frac{1}{h} \Delta h u_{n}=\frac{1}{g h_{N}} \Delta h_{N} v_{n}=\frac{1}{g} \Delta^{h_{N}} v_{n}$, it follows that $v_{n}$ is the minimal positive solution to $\Delta^{h_{N}} v_{n}=-g I_{[n-1, n]}$ with the Neumann boundary condition on $\Gamma_{N}$ and with the Dirichlet boundary condition on $\Gamma_{D}$ and on $\{r=1\}$. From this it follows that

$$
u_{n}(r, y)=\frac{v_{n}}{g}(r, y)=\frac{1}{g(r, y)} E_{r, y}^{h_{N}} \int_{0}^{\tau_{1} \wedge \sigma} g(r(s), y(s)) I_{[n-1, n]}(r(s)) d s .
$$

Thus,

$$
u_{n}(n, y) \leq \frac{1}{g(n, y)} \sup _{\substack{r \in[n-1, n] \\ y^{\prime}}} g\left(r, y^{\prime}\right) E_{n, y}^{h_{N}} \int_{0}^{\tau_{1} \wedge \sigma} I_{[n-1, n]}(r(s)) d s .
$$

Recalling from (1.16) that $h(r, \pm 1)$ does not vanish if $r \notin \bigcup_{m=1}^{\infty}\left[a_{m}, b_{m}\right] \subset$ $\bigcup_{m=1}^{\infty}\left[m+\frac{1}{4}, m+\frac{3}{4}\right]$, it follows by Harnack's inequality that

$$
\sup _{\substack{r \in[n-1, n] \\ y^{\prime}}} \frac{h\left(r, y^{\prime}\right)}{h(n, y)}<\infty .
$$


From (3.11), (3.12) and the definitions of $h, h_{N}$, and $g$, it follows that there exists a constant $c>0$ such that

$$
u_{n}(n, y) \leq c E_{n, y}^{h_{N}} \int_{0}^{\tau_{1} \wedge \sigma} I_{[n-1, n]}(r(s)) d s .
$$

We leave it to the reader to check that

$$
\hat{C} \equiv \sup _{n} E_{n, y}^{h_{N}} \int_{0}^{\tau_{n+1} \wedge \tau_{1}} I_{[n-1, n]}(r(s)) d s<\infty .
$$

This is done via a direct calculation since $F_{n}(r) \equiv E_{r, y}^{h_{N}} \int_{0}^{\tau_{n+1} \wedge \tau_{1}} I_{[n-1, n]}(r(s)) d s$ satisfies $\left(\frac{1}{2} \frac{d^{2}}{d r^{2}}+\frac{3-d}{2 r} \frac{d}{d r}\right) F_{n}(r)=-I_{[n-1, n]}(r)$, for $r \in(1, n+1)$ and $F_{n}(1)=$ $F_{n}(n+1)=0$. Let

$$
\gamma_{n}=\inf _{y} P_{n+1, y}^{h_{N}}\left(\sigma<\tau_{n}\right) .
$$

It follows from (3.13), (3.14), (3.15) and the strong Markov property that

$$
u_{n}(n, y) \leq \hat{C} \sum_{m=1}^{\infty} m\left(1-\gamma_{n}\right)^{m-1} \gamma_{n}=\frac{\hat{C}}{\gamma_{n}} .
$$

We will prove the following lemma.

Lemma 7. For any $q>1$, there exists a constant $c_{q}>0$ such that

$$
\gamma_{n}>c_{q}\left(\left|\log \rho_{n, q n}^{-}\right|^{-\frac{1}{2}} \wedge \frac{n}{\left|\log \rho_{n, q n}^{-}\right|}\right),
$$

where $\rho_{n, q n}^{-}$is as in the statement of Theorem $2^{\prime}$.

It follows from Lemma 7 and (3.16) that (3.10) will hold if (1.12) holds. Thus, the proof of (3.1) will be complete when we prove Lemma 7 .

Recall the definition of $\left\{\nu_{n}\right\}_{n=0}^{\infty}$ from the beginning of section 2. Define

$$
W_{n}=j \text {, if } \nu_{j}=\tau_{n} .
$$

Under $P_{m}^{h_{N}}$, for $m$ an integer, $W_{n}$ counts the number of times the process $r(t)$ upcrosses or downcrosses an interval of the form $[j, j+1]$ before reaching $n$.

Proof of Lemma 7. In the calculations that follow, $c$ will denote a positive constant whose value may change from line to line. For $q>1$, we write

$$
\begin{aligned}
& P_{n+1, y}^{h_{N}}\left(\sigma<\tau_{n}\right) \geq P_{n+1, y}^{h_{N}}\left(\sigma<\tau_{n} ; \sup _{0 \leq t \leq \tau_{n}} r(t)<q n\right) \\
& =\sum_{m=1}^{\infty} P_{n+1, y}^{h_{N}}\left(\sigma<\tau_{n} \mid W_{n}=m, \sup _{0 \leq t \leq \tau_{n}} r(t)<q n\right) P_{n+1}^{h_{N}}\left(W_{n}=m, \sup _{0 \leq t \leq \tau_{n}} r(t)<q n\right),
\end{aligned}
$$

and

$$
\begin{aligned}
& P_{n+1, y}^{h_{N}}\left(\tau_{n}<\sigma \mid W_{n}=m, \sup _{0 \leq t \leq \tau_{n}} r(t)<q n\right) \\
& =E_{n+1, y}^{h_{N}} P_{n+1, y}^{h_{N}}\left(\tau_{n}<\sigma \mid W_{n}=m, \sup _{0 \leq t \leq \tau_{n}} r(t)<q n, r\left(\nu_{j}\right), j=1, \ldots, m-1\right) .
\end{aligned}
$$


Similar to (2.18), for any sequence $\left\{r_{j}\right\}_{j=0}^{m}$ satisfying $r_{0}=n+1,\left|r_{j+1}-r_{j}\right|=$ $1, r_{m}=n$, and $r_{j} \in\{n+1, \ldots,[q n]\}$, for $j=1, \ldots, m-1$, we have

$$
\begin{aligned}
& P_{n+1, y}^{h_{N}}\left(\tau_{n}<\sigma \mid W_{n}=m, \sup _{0 \leq t \leq \tau_{n}} r(t)<q n,\left\{r\left(\nu_{j}\right)\right\}_{j=1}^{m-1}=\left\{r_{j}\right\}_{j=1}^{m-1}\right) \\
& =\prod_{j=0}^{m-1} P_{r_{j}, y\left(\nu_{j}\right)}^{h_{N}}\left(\tau_{r_{j}-1} \wedge \tau_{r_{j}+1}<\sigma \mid \tau_{r_{j}-1} \wedge \tau_{r_{j}+1}=\tau_{r_{j+1}}\right) .
\end{aligned}
$$

Using Lemma 1 for the first inequality below, and the condition on the sequence $\left\{r_{j}\right\}$ for the second inequality, we have

$$
\begin{aligned}
& P_{r_{j}, y}^{h_{N}}\left(\tau_{r_{j}-1} \wedge \tau_{r_{j}+1}<\sigma \mid \tau_{r_{j}-1} \wedge \tau_{r_{j}+1}=\tau_{r_{j+1}}\right) \leq 1-\frac{c_{1}}{\left|\log \rho_{r_{j}}\right|} \\
& \leq 1-\frac{c}{\left|\log \rho_{n, q n}^{-}\right|} .
\end{aligned}
$$

From (3.18)-(3.20) we have

$$
P_{n+1, y}^{h_{N}}\left(\tau_{n}<\sigma \mid W_{n}=m, \sup _{0 \leq t \leq \tau_{n}} r(t)<q n\right) \leq\left(1-\frac{c}{\left|\log \rho_{n, q n}^{-}\right|}\right)^{m} .
$$

Using (3.21) in (3.17), we obtain

$$
P_{n+1, y}^{h_{N}}\left(\sigma<\tau_{n}\right) \geq \sum_{m=1}^{\infty}\left(1-\left(1-\frac{c}{\left|\log \rho_{n, q n}^{-}\right|}\right)^{m}\right) P_{n+1}^{h_{N}}\left(W_{n}=m, \sup _{0 \leq t \leq \tau_{n}} r(t)<q n\right) .
$$

Using the summation by parts formula,

$$
\sum_{m=1}^{\infty} p_{m} q_{m}=p_{1} Q_{1}+\sum_{m=2}^{\infty}\left(p_{m}-p_{m-1}\right) Q_{m}
$$

where $Q_{m}=\sum_{k=m}^{\infty} q_{k}$, and where the condition $\lim _{n \rightarrow \infty} p_{n} Q_{n+1}=0$ holds, we obtain from (3.22) and (3.15) that

$$
\left.\gamma_{n} \geq \frac{c}{\left|\log \rho_{n, q n}^{-}\right|}\right) \sum_{m=1}^{\infty}\left(1-\frac{c}{\left|\log \rho_{n, q n}^{-}\right|}\right)^{m-1} P_{n+1}^{h_{N}}\left(W_{n} \geq m, \sup _{0 \leq t \leq \tau_{n}} r(t)<q n\right) .
$$

We also have for some $c_{1}>0$,

$$
\begin{aligned}
& \sum_{m=1}^{\infty}\left(1-\frac{c}{\left|\log \rho_{n, q n}^{-}\right|}\right)^{m-1} P_{n+1}^{h_{N}}\left(W_{n} \geq m, \sup _{0 \leq t \leq \tau_{n}} r(t)<q n\right) \\
& \geq c_{1} \sum_{m=1}^{\left[\left|\log \rho_{n, q n}^{-}\right|\right]} P_{n+1}^{h_{N}}\left(W_{n} \geq m, \sup _{0 \leq t \leq \tau_{n}} r(t)<q n\right) \\
& =c_{1} E_{n+1}^{h_{N}}\left(W_{n} \wedge\left[\left|\log \rho_{n, q n}^{-}\right|\right]\right) 1_{\left\{\sup _{0 \leq t \leq \tau_{n}} r(t)<q n\right\} .}
\end{aligned}
$$

Now we will show that

$$
E_{n+1}^{h_{N}}\left(W_{n} \wedge\left[\left|\log \rho_{n, q n}^{-}\right|\right]\right) 1_{\left\{\sup _{0 \leq t \leq \tau_{n}} r(t)<q n\right\}} \geq c\left|\log \rho_{n, q n}^{-}\right|^{\frac{1}{2}} \wedge n .
$$

Lemma 7 will then follow from (3.23)-(3.25). 

that

To prove (3.25), let $M_{n}=\left[\left|\log \rho_{n, q n}^{-}\right|^{\frac{1}{2}} \wedge \frac{q-1}{2} n\right]$. Then (3.25) will hold if we show

$$
E_{n+1}^{h_{N}}\left(W_{n} \wedge M_{n}^{2}\right) 1_{\left\{\sup _{0 \leq t \leq \tau_{n}} r(t)<q n\right\}} \geq c M_{n} .
$$

Note that there exists a constant $c>0$, independent of $n$ such that

$$
P_{n+1}^{h_{N}}\left(\tau_{n+M_{n}}<\tau_{n}\right) \geq \frac{c}{M_{n}} .
$$

Indeed (3.27) can be proved by a direct calculation since the left-hand side is equal to $f_{n}(n+1)$ where $f_{n}$ satisfies $\frac{1}{2} f_{n}^{\prime \prime}+\frac{3-d}{2 r} f_{n}^{\prime}=0$ for $r \in\left(n, n+M_{n}\right)$ and $f(n)=$ $0, f\left(n+M_{n}\right)=1$. We also claim that there exists a $c>0$, independent of $n$, such that

$$
P_{n+M_{n}}^{h_{N}}\left(W_{n} \geq M_{n}^{2}, \sup _{0 \leq t \leq \tau_{n}} r(t)<q n\right) \geq c .
$$

It's easy to see from the strong Markov property and the definition of $W_{n}$ that if (3.27) and (3.28) hold, then (3.26) holds. Thus, it remains to show (3.28).

To show (3.28), we note that, by Brownian scaling, it follows that, for each $L>0$, there exists a $c(L)>0$, independent of $n$, such that

$$
P_{n+M_{n}}^{h_{N}}\left(\tau_{n} \geq L M_{n}^{2}, \tau_{n}<\tau_{n+2 M_{n}}\right) \geq c(L) .
$$

Note that under $P_{m}^{h_{N}}$, for $m$ an integer greater than $n, \tau_{n}$ can be represented by

$$
\tau_{n}=\sum_{j=1}^{W_{n}}\left(\nu_{j}-\nu_{j-1}\right) .
$$

Since by assumption, $n+2 M_{n}<q n$, it follows from (3.30) that

$$
\begin{aligned}
& P_{n+M_{n}}^{h_{N}}\left(\tau_{n} \geq L M_{n}^{2}, \tau_{n}<\tau_{n+2 M_{n}}\right) \\
& \leq P_{n+M_{n}}^{h_{N}}\left(W_{n} \geq M_{n}^{2}, \sup _{0 \leq t \leq \tau_{n}} r(t)<q n\right) \\
& +P_{n+M_{n}}^{h_{N}}\left(\sum_{j=1}^{W_{n}}\left(\nu_{j}-\nu_{j-1}\right) \geq L M_{n}^{2}, W_{n}<M_{n}^{2}, \sup _{0 \leq t \leq \tau_{n}} r(t)<q n\right) .
\end{aligned}
$$

Letting $A_{n}$ denote the event $\left\{W_{n}<M_{n}^{2}, \sup _{0 \leq t \leq \tau_{n}} r(t)<q n\right\}$, we rewrite the second term on the right-hand side of (3.31) as

$$
\begin{aligned}
& P_{n+M_{n}}^{h_{N}}\left(\sum_{j=1}^{W_{n}}\left(\nu_{j}-\nu_{j-1}\right) \geq L M_{n}^{2}, W_{n}<M_{n}^{2}, \sup _{0 \leq t \leq \tau_{n}} r(t)<q n\right) \\
& =E_{n+M_{n}}^{h_{N}}\left(P_{n+M_{n}}^{h_{N}}\left(\sum_{j=1}^{W_{n}}\left(\nu_{j}-\nu_{j-1}\right) \geq L M_{n}^{2} \mid A_{n}\right) ; A_{n}\right) .
\end{aligned}
$$

Now for any $m<M_{n}^{2}$, and any sequence $\left\{r_{j}\right\}_{j=0}^{m}$ satisfying $r_{0}=n+M_{n}, r_{m}=$ $n, n<r_{j}<q n$, for $j \in\{1,2, \ldots, m-1\}$, and $\left|r_{j}-r_{j-1}\right|=1$, the random variables $\left\{\nu_{k}-\nu_{k-1}\right\}_{k=1}^{m}$ under $P_{n+M_{n}}^{h_{N}}\left(\cdot \mid W_{n}=m,\left\{r\left(\nu_{j}\right)\right\}_{j=1}^{m-1}=\left\{r_{j}\right\}_{j=1}^{m-1}\right)$ are independent and $\nu_{k+1}-\nu_{k}$ is distributed like $\tau_{r_{k+1}}$ under $P_{r_{k}}^{h_{N}}\left(\cdot \mid \tau_{r_{k}-1} \wedge \tau_{r_{k}+1}=\tau_{r_{k+1}}\right)$.

We now need the following fact:

There exists a $\lambda_{0}>0$ such that

$$
\psi(\lambda) \equiv \sup _{n} E_{n}^{h_{N}}\left(\exp \left(\lambda\left(\tau_{n-1} \wedge \tau_{n+1}\right)\right) \mid \tau_{n \pm 1}<\tau_{n \mp 1}\right)<\infty \text {, for } \lambda \leq \lambda_{0} .
$$


To show (3.33), we note that standard methods, which any reader who has followed this article up until here should be able to reproduce without much difficulty, show that there exists a $\lambda_{0}>0$ such that the principal eigenvalue for the operator $\frac{1}{2} \frac{d^{2}}{d r^{2}}+\frac{3-d}{2 r} \frac{d}{d r}$ in $[n-1, n+1]$ with the Dirichlet boundary condition at $n-1$ and $n+1$ is larger than $\lambda_{0}$ for all $n$, and that $\sup _{\lambda<\lambda_{0}, n} E_{n}^{h_{N}} \exp \left(\lambda\left(\tau_{n-1} \wedge \tau_{n+1}\right)\right)<$ $\infty$. By the strong Markov property, $E_{n}^{h_{N}} \exp \left(\lambda\left(\tau_{n-1} \wedge \tau_{n+1}\right) \mid \tau_{n+1}<\tau_{n-1}\right)=$ $\frac{1}{f_{n}(n)} E_{n}^{h_{N}}\left(\exp \left(\lambda\left(\tau_{n-1} \wedge \tau_{n+1}\right)\right) ; \tau_{n+1}<\tau_{n-1}\right)$, where, for $r \in[n-1, n+1]$,

$$
f_{n}(r)=P_{r}^{h_{N}}\left(\tau_{n+1}<\tau_{n-1}\right)=\frac{r^{d-2}-(n-1)^{d-2}}{(n+1)^{d-2}-(n-1)^{d-2}} .
$$

A similar formula holds of course when the terms $\tau_{n+1}$ and $\tau_{n-1}$ are interchanged. This proves $(3.33)$.

Using (3.32), (3.33), the claims in the text between (3.32) and (3.33), and Chebyshev's inequality, we have, for $\lambda \in\left(0, \lambda_{0}\right)$ and $m \leq M_{n}^{2}$,

$$
\begin{aligned}
& P_{n+1}^{h_{N}}\left(\sum_{j=1}^{W_{n}}\left(\nu_{j}-\nu_{j-1}\right) \geq L M_{n}^{2} \mid W_{n}=m,\left\{r\left(\nu_{j}\right)\right\}_{j=1}^{m-1}=\left\{r_{j}\right\}_{j=1}^{m-1}\right) \\
& \leq \exp \left(-\lambda L M_{n}^{2}\right) \psi^{m}(\lambda) \leq \exp \left(-\lambda L M_{n}^{2}\right) \psi^{M_{n}^{2}}(\lambda)
\end{aligned}
$$

Choose $L$ sufficiently large so that $\gamma \equiv \exp (-\lambda L) \psi(\lambda)<1$. Using this in (3.34), and using (3.31) and (3.32), we conclude that

$$
\begin{aligned}
& P_{n+M_{n}}^{h_{N}}\left(\tau_{n} \geq L M_{n}^{2}, \tau_{n}<\tau_{n+2 M_{n}}\right) \\
& \leq P_{n+M_{n}}^{h_{N}}\left(W_{n} \geq M_{n}^{2}, \sup _{0 \leq t \leq \tau_{n}} r(t)<q n\right)+\gamma^{M_{n}^{2}} .
\end{aligned}
$$

Since the left-hand side of (3.28) is obviously positive for all $n,(3.28)$ now follows from (3.29) and (3.35). This completes the proof of Lemma 7 and thus also of (3.1).

Proof of (3.2) under the assumption that (1.13) holds. First of all, we note that by the strong Markov property it's enough to prove (3.2) with $r \rightarrow \infty$ replaced by $n \rightarrow \infty$, where $n$ represents the natural numbers. Using the strong Markov property again, it follows that, for any integer $n>1$, and any sequence $\left\{y_{j}\right\}_{j=1}^{n-1}$, the random variables

$$
\left\{\int_{\tau_{j+1}}^{\tau_{j}} \frac{1}{r^{2}(s)} d s\right\}_{j=1}^{n-1}
$$

are independent under $P_{n, y}^{h}\left(\cdot \mid y\left(\tau_{j}\right)=y_{j}, j=1, \ldots, n-1\right)$. Since

$$
\int_{0}^{\tau_{1}} \frac{1}{r^{2}(s)} d s=\sum_{j=1}^{n-1} \int_{\tau_{j+1}}^{\tau_{j}} \frac{1}{r^{2}(s)} d s
$$

under $P_{n, y}^{h}\left(\cdot \mid y\left(\tau_{j}\right)=y_{j}, j=1, \ldots, n-1\right)$, and since for any $K>0, \int_{\tau_{j+1}}^{\tau_{j} \wedge K j^{2}} \frac{1}{r^{2}(s)} d s \leq$ $K$, (3.2) will follow easily from Proposition 1 if we show that, for some $K>0$ and for any sequence $\left\{y_{j}\right\}_{j=1}^{\infty}$,

$$
\lim _{n \rightarrow \infty} E_{n, y_{n}}^{h}\left(\sum_{j=1}^{n-1} \int_{\tau_{j+1}}^{\tau_{j} \wedge K j^{2}} \frac{1}{r^{2}(s)} d s \mid y\left(\tau_{j}\right)=y_{j}, j=1, \ldots, n-1\right)=\infty .
$$


Rewriting the above requirement using the strong Markov property, and making the expressions smaller by replacing $\tau_{j}$ in the upper limit of the integral by $\tau_{j+\frac{1}{16}}$, it follows that (3.36) will hold if we show that

$$
\left.\sum_{n=1}^{\infty} E_{n+1, y_{n+1}}^{h}\left(\int_{0}^{K n^{2} \wedge \tau_{n+\frac{1}{16}}} \frac{1}{r^{2}(s)} d s\right) \mid y\left(\tau_{n}\right)=y_{n}\right)=\infty .
$$

Using the strong Markov property, we have, for any $A \subset[-1,1]$,

$$
\begin{aligned}
& \left.E_{n+1, y_{n+1}}^{h}\left(\int_{0}^{K n^{2} \wedge \tau_{n+\frac{1}{16}}} \frac{1}{r^{2}(s)} d s\right) \mid y\left(\tau_{n}\right) \in A\right) \\
& =\frac{E_{n+1, y_{n+1}}^{h}\left(\int_{0}^{K n^{2} \wedge \tau_{n+\frac{1}{16}}} \frac{1}{r^{2}(s)} d s\right) P_{n+\frac{1}{16}, y\left(\tau_{n+\frac{1}{16}}\right)}^{h}\left(y\left(\tau_{n}\right) \in A\right)}{P_{n+1, y_{n+1}}^{h}\left(y\left(\tau_{n}\right) \in A\right)} .
\end{aligned}
$$

We will now show that there exist positive constants $c_{1}, c_{2}$, independent of $n \geq 1$ and $y \in[-1,1]$, such that

$$
c_{1} \leq \frac{d P_{n+\frac{1}{16}, y}^{h}\left(y\left(\tau_{n}\right) \in \cdot\right)}{d P_{n+1, y_{n+1}}^{h}\left(y\left(\tau_{n}\right) \in \cdot\right)} \leq c_{2}
$$

Let $w \geq 0$ denote a nonnegative, bounded continuous test function, and define $f_{n}(r, y)=E_{r, y}^{h} w\left(y\left(\tau_{n}\right)\right)$, for $r>n$ and $y \in[-1,1]$. Then (3.39) will follow if we show that there exist positive constants $c_{1}, c_{2}$, independent of $n \geq 1, y \in(-1,1)$, and $w$, such that

$$
c_{1} \leq \frac{f_{n}\left(n+\frac{1}{16}, y\right)}{f_{n}\left(n+1, y_{n+1}\right)} \leq c_{2} .
$$

To show (3.40), let $F_{n}(r, y)=E_{r, y}^{h_{N}}\left(w\left(y\left(\tau_{n}\right)\right) ; \tau_{n}<\sigma\right)$, for $r>n$ and $y \in[-1,1]$. Also, let $g=\frac{h}{h_{N}}$ as we did after (3.10). Note that $F_{n}$ and $g$ are $\Delta^{h_{N}}$-harmonic for $r>n$ and $y \in[-1,1]$ and satisfy the Neumann boundary condition on $\Gamma_{N}$ and the Dirichlet boundary condition on $\Gamma_{D}$. Since the coefficients of $\Delta^{h_{N}}$ are uniformly bounded for $r>1$ and $y \in[-1,1]$, and since by (1.16), $\Gamma_{D} \cap$ $\left\{r \in\left[n-\frac{1}{4}, n+\frac{5}{4}\right]\right\} \subset\left\{r \in\left(n+\frac{1}{4}, n+\frac{3}{4}\right)\right\}$, it follows by Harnack's inequality that there exist positive constants $c_{1}, c_{2}$, independent of $n \geq 1$ and $y \in(-1,1)$, such that $c_{1} \leq \frac{F_{n}\left(n+\frac{1}{16}, y\right)}{F_{n}\left(n+1, y_{n+1}\right)} \leq c_{2}$, and such that $c_{1} \leq \frac{g(r, y)}{g\left(n, y_{n}\right)} \leq c_{2}$, for $r=n+1$ or $r=n+\frac{1}{16}$. These inequalities along with the representation

$$
f_{n}(r, y)=E_{r, y}^{h} w\left(y\left(\tau_{n}\right)\right)=\frac{E_{r, y}^{h_{N}}\left(w\left(y\left(\tau_{n}\right)\right) g\left(n, y\left(\tau_{n}\right)\right) ; \tau_{n}<\sigma\right)}{g(r, y)}
$$

complete the proof of (3.40), and thus of (3.39).

From (3.38) and (3.39), it follows that we can remove the conditioning in (3.37); that is, (3.37) is equivalent to

$$
\sum_{n=1}^{\infty} E_{n+1, y}^{h} \int_{0}^{K n^{2} \wedge \tau_{n+\frac{1}{16}}} \frac{1}{r^{2}(s)} d s=\infty
$$


Define $G=\left(\bigcup_{n=1}^{\infty}\left[n+\frac{1}{16}, n+\frac{1}{8}\right]\right) \times(-1,1)$. Let $q>1$. To show that (3.41) holds, it of course suffices to show that

$$
\sum_{n=1}^{\infty} E_{n+1, y}^{h} \int_{0}^{K n^{2} \wedge \tau_{q n} \wedge \tau_{n+\frac{1}{16}}} \frac{1}{r^{2}(s)} 1_{G}(r(s)) d s=\infty,
$$

or equivalently, that

$$
\sum_{n=1}^{\infty} \frac{U_{n}(n+1, y)}{n^{2}}=\infty
$$

where

$$
U_{n}(r, y)=E_{r, y}^{h} \int_{0}^{K n^{2} \wedge \tau_{q n} \wedge \tau_{n+\frac{1}{16}}} 1_{G}(r(s)) d s, r \in\left(n+\frac{1}{16}, q n\right), y \in(-1,1) .
$$

Now $U_{n}(r, y)$ is equal to $\hat{U}_{n}\left(r, y, K n^{2}\right)$, where $\hat{U}_{n}(r, y, t)$ satisfies the parabolic equation

$$
\begin{aligned}
& \frac{\partial \hat{U}_{n}}{\partial t}=\frac{1}{2} \Delta^{h} \hat{U}_{n}+1_{G}, \quad r \in\left(n+\frac{1}{16}, q n\right), y \in(-1,1), \\
& \hat{U}_{n}\left(n+\frac{1}{16}, y, t\right)=\hat{U}_{n}(q n, y, t)=0, \quad y \in[-1,1], t>0, \\
& \hat{U}_{n}(r, y, 0)=0, \quad r \in\left(n+\frac{1}{16}, q n\right), y \in[-1,1] .
\end{aligned}
$$

Letting $g=\frac{h}{h_{n}}$ and $\hat{V}_{n}=g \hat{U}_{n}$, it follows by the argument appearing between (3.10) and (3.11) that $\hat{V}_{n}$ satisfies $\frac{\partial \hat{V}_{n}}{\partial t}=\frac{1}{2} \Delta^{h_{N}} \hat{V}_{n}+g 1_{G}, \hat{V}_{n}$ satisfies the Dirichlet boundary condition at $r=n+\frac{1}{16}$, at $r=q n$, and on $\Gamma_{D}, \hat{V}_{n}$ satisfies the Neumann boundary condition on $\Gamma_{N}$, and $\hat{V}_{n}$ vanishes at $t=0$. Thus, the type of analysis used between (3.10) and (3.11) shows that

$$
U_{n}(r, y)=\hat{U}_{n}\left(r, y, K n^{2}\right)=\frac{1}{g(r, y)} E_{r, y}^{h_{N}} \int_{0}^{K n^{2} \wedge \tau_{q n} \wedge \tau_{n+\frac{1}{16}} \wedge \sigma} g(r(s), y(s)) 1_{G}(r(s)) d s .
$$

To proceed, we need the following lemma.

Lemma 8. Let $M_{n}=\left[\left(\rho_{n, q n}^{+}\right)^{\frac{1}{2}} \wedge \frac{(q-1)}{2} n\right]$, where $\rho_{n, q n}^{+}$is as in the statement of Theorem $2^{\prime}$, and let $G_{n}=\left(\bigcup_{m=n}^{n+M_{n}}\left[m+\frac{1}{16}, m+\frac{1}{8}\right]\right) \times(-1,1)$. Then there exists a $c>0$, independent of $n$, such that

$$
\inf _{(r, y) \in G_{n}} g(r, y) \geq c \sup _{y} g(n, y) .
$$

Proof. Recall from its definition that $g(r, y)=P_{r, y}^{h_{N}}\left(\tau_{1}<\sigma\right)$. Thus, by the strong Markov property (or the maximum principle), $\sup _{y} g(r, y)$ is decreasing in $r$. Also, by Harnack's inequality, (1.16) and the definition of $G_{n}$, there exists a $c>0$ such that $\inf _{y} g(r, y) \geq c \sup _{y} g(r, y)$, for $r \in \bigcup_{n=1}^{\infty} G_{n}$. Thus, it suffices to prove the estimate

$$
\sup _{y} g\left(n+M_{n}, y\right) \geq c \inf _{y} g(n, y) .
$$


By the strong Markov property, we have

$$
g\left(n+M_{n}, y\right)=E_{n+M_{n}, y}^{h_{N}}\left(g\left(n, y\left(\tau_{n}\right)\right) ; \tau_{n}<\sigma\right) .
$$

Thus, (3.44) will follow from (3.45) if we show that

$$
\inf _{n \geq 2} P_{n+M_{n}, y}^{h_{N}}\left(\tau_{n}<\sigma\right)>0 .
$$

The same type of argument that showed (3.21) shows that

$$
P_{n+M_{n}, y}^{h_{N}}\left(\tau_{n}<\sigma \mid W_{n}=m, \sup _{0 \leq t \leq \tau_{n}} r(t)<q n\right) \geq\left(1-\frac{c}{\left|\log \rho_{n, q n}^{+}\right|}\right)^{m} .
$$

Thus, recalling the definition of $M_{n},(3.46)$ will certainly hold if we show that there exists a $c>0$ independent of $n$ such that

$$
P_{n+M_{n}, y}^{h_{N}}\left(W_{n} \leq M_{n}^{2}, \sup _{0 \leq t \leq \tau_{n}} r(t)<q n\right) \geq c .
$$

The proof of (3.47) is very similar to the proof of (3.28). Indeed, by Brownian scaling, (3.29) still holds when $\tau_{n} \geq L M_{n}^{2}$ is replaced by $\tau_{n} \leq L M_{n}^{2}$. Also, (3.31) holds with the inequalities $\tau_{n} \geq L M_{n}^{2}, W_{n} \geq M_{n}^{2}, \sum_{j=1}^{W_{n}}\left(\nu_{j}-\nu_{j-1}\right) \geq L M_{n}^{2}$, and $W_{n}<M_{n}^{2}$ all reversed. Now use a parallel argument to the one used after (3.31), this time employing Chebyshev's inequality with $\lambda<0$ and with $L>0$ sufficiently small. We leave the rest of the details to the reader.

From Lemma 8 and (3.43) we obtain

$$
U_{n}(n+1, y) \geq c E_{n+1, y}^{h_{N}} \int_{0}^{K n^{2} \wedge \tau_{q n} \wedge \tau_{n+\frac{1}{16}} \wedge \sigma} 1_{G_{n}}(r(s)) d s .
$$

Recalling the definition of $M_{n}$ in Lemma 8, and noting that $n+M_{n}<q n$, we then have

$$
U_{n}(n+1, y) \geq c E_{n+1, y}^{h_{N}} \int_{0}^{K n^{2} \wedge \tau_{n+M_{n}} \wedge \tau_{n+\frac{1}{16}} \wedge \sigma} 1_{G}(r(s)) d s .
$$

Recall again the definition of $\nu_{j}$ from the beginning of section 2. Define $\hat{W}_{n}$ as follows:

$$
\hat{W}_{n}=\inf \left\{j: \nu_{j+1}>\tau_{n+\frac{1}{16}} \text { or } \nu_{j}=\tau_{n+M_{n}}\right\} .
$$

Thus, under $P_{n+1}^{h_{N}}, \hat{W}_{n}$ counts the number of times $r(t)$ upcrosses or downcrosses intervals of the form $[m, m+1], m \in\left\{n+1, \ldots, n+M_{n}-1\right\}$ before hitting $n+\frac{1}{16}$ or $n+M_{n}$. Now let $\hat{Y}_{0}=\int_{\nu_{\hat{W}_{n}}}^{\tau_{n+\frac{1}{16}} \wedge \tau_{n+M_{n}}} 1_{G}(r(s)) d s$, and if $\hat{W}_{n} \geq 1$, let

$$
\hat{Y}_{j}=\int_{\nu_{j-1}}^{\nu_{j}} 1_{G}(r(s)) d s, j=1, \ldots, \hat{W}_{n} .
$$

Similarly, let $Y_{0}=\tau_{n+\frac{1}{16}} \wedge \tau_{n+M_{n}}-\nu_{\hat{W}_{n}}$, and if $\hat{W}_{n} \geq 1$, let

$$
Y_{j}=\nu_{j}-\nu_{j-1}, j=1, \ldots, \hat{W}_{n} .
$$

Note that

$$
\int_{0}^{\tau_{n+M_{n}} \wedge \tau_{n+\frac{1}{16}}} 1_{G}(r(s)) d s=\sum_{j=0}^{\hat{W}_{n}} \hat{Y}_{j} \text { and } \tau_{n+M_{n}} \wedge \tau_{n+\frac{1}{16}}=\sum_{j=0}^{\hat{W}_{n}} Y_{j} \text { a.s. } P_{n+1}^{h_{N}} .
$$


Thus we have the inequality

$$
\begin{aligned}
& E_{n+1, y}^{h_{N}} \int_{0}^{K n^{2} \wedge \tau_{n+M_{n}} \wedge \tau_{n+\frac{1}{16}} \wedge \sigma} 1_{G}(r(s)) d s \\
& \geq E_{n+1, y}^{h_{N}}\left(1_{\tau_{n+\frac{1}{16}} \wedge \tau_{n+M_{n}}<\sigma}\right)\left(1_{\sum_{j=0}^{\hat{W}_{n}} Y_{j} \leq K n^{2}} \sum_{j=0}^{\hat{W}_{n}} \hat{Y}_{j}\right) .
\end{aligned}
$$

Let $l$ be a positive integer and let $\left\{r_{j}\right\}_{j=0}^{l}$ be a sequence satisfying $r_{0}=n+$ $1, r_{m}=n+1$ or $n+M_{n},\left|r_{j+1}-r_{j}\right|=1, r_{j} \in\left\{n+1, \ldots, n+M_{n}-1\right\}$, for $j=1, \ldots, m-1$, and let $\left\{y_{j}\right\} \in[-1,1]$. Let $A_{n, l}=\left\{\tau_{n+\frac{1}{16}} \wedge \tau_{n+M_{n}}<\sigma, \hat{W}_{n}=l, r\left(\nu_{j}\right)=r_{j}, y\left(\nu_{j}\right)=\right.$ $\left.y_{j}, j=1, \ldots, l\right\}$. (We suppress the dependence of $A_{n, l}$ on all the other variables.) Note that under $P_{n+1, y}^{h_{N}}\left(\cdot \mid A_{n, l}\right)$, the random vectors $\left\{\left(Y_{j}, \hat{Y}_{j}\right)\right\}_{j=0}^{l}$ are independent. To estimate the right-hand side of (3.49), we will need the following lemma whose long proof we postpone until the completion of the proof of the theorem.

Lemma 9. . There exists a $c>0$, independent of $n, l$ and $k$, such that

$$
\left.E_{n+1, y}^{h_{N}}\left(1_{\sum_{j=0}^{\hat{W}_{n}} Y_{j} \leq K n^{2}} \hat{Y}_{k}\right) \mid A_{n, l}\right) \geq c, \text { for } k \leq l \leq n^{2} .
$$

Using (3.50), we have

$$
\begin{aligned}
& E_{n+1, y}^{h_{N}}\left(1_{\tau_{n+\frac{1}{16}} \wedge \tau_{n+M_{n}}<\sigma}\left(1_{\sum_{j=0}^{\hat{W}_{n}} Y_{j} \leq K n^{2}} \sum_{j=0}^{\hat{W}_{n}} \hat{Y}_{j}\right) \mid A_{n, l}\right) \\
& =E_{n+1, y}^{h_{N}}\left(1_{\sum_{j=0}^{\hat{W}_{n}} Y_{j} \leq K n^{2}} \sum_{j=0}^{\hat{W}_{n}} \hat{Y}_{j} \mid A_{n, l}\right) \geq c l, \text { for } l \leq n^{2} .
\end{aligned}
$$

Thus,

$$
\begin{aligned}
& E_{n+1, y}^{h_{N}}\left(1_{\tau_{n+\frac{1}{16}}} \wedge \tau_{n+M_{n}}<\sigma\right)\left(1_{\sum_{j=0}^{\hat{W}_{n}} Y_{j} \leq K n^{2}} \sum_{j=0}^{\hat{W}_{n}} \hat{Y}_{j}\right) \\
& \geq E_{n+1, y}^{h_{N}}\left(\hat{W}_{n} \wedge n^{2} ; \tau_{n+\frac{1}{16}} \wedge \tau_{n+M_{n}}<\sigma\right) .
\end{aligned}
$$

Recalling that $n+M_{n}<q n$, we note that the same type of argument used to show (3.21) shows that

$$
P_{n+1, y}^{h_{N}}\left(\tau_{n+\frac{1}{16}} \wedge \tau_{n+M_{n}}<\sigma \mid \hat{W}_{n}=k\right) \geq\left(1-\frac{c}{\left|\log \rho_{n, q n}^{+}\right|}\right)^{k} .
$$

Thus, writing

$$
\begin{aligned}
& P_{n+1, y}^{h_{N}}\left(\hat{W}_{n}=k, \tau_{n+\frac{1}{16}} \wedge \tau_{n+M_{n}}<\sigma\right) \\
& =P_{n+1, y}^{h_{N}}\left(\tau_{n+\frac{1}{16}} \wedge \tau_{n+M_{n}}<\sigma \mid \hat{W}_{n}=k\right) P_{n+1, y}^{h_{N}}\left(\hat{W}_{n}=k\right),
\end{aligned}
$$

it follows from (3.52) that, for some $c>0$,

$$
E_{n+1, y}^{h_{N}}\left(\hat{W}_{n} \wedge n^{2} ; \tau_{n+\frac{1}{16}} \wedge \tau_{n+M_{n}}<\sigma\right) \geq c E_{n+1, y}^{h_{N}}\left(\hat{W}_{n} \wedge n^{2} \wedge\left|\log \rho_{n, q n}^{+}\right|\right) .
$$

A proof just like the proof of (3.25) shows that

$$
E_{n+1}^{h_{N}}\left(\hat{W}_{n} \wedge n^{2} \wedge\left|\log \rho_{n, q n}^{+}\right|\right) \geq c\left|\log \rho_{n, q n}^{+}\right|^{\frac{1}{2}} \wedge n .
$$


From (3.48), (3.49), (3.51), (3.53) and (3.54), we have

$$
U(n+1, y) \geq c\left|\log \rho_{n, q n}^{+}\right|^{\frac{1}{2}} \wedge n .
$$

From (3.55) and condition (1.13) in part ii of the theorem, we conclude that (3.42) holds, thereby completing the proof of (3.2).

We now return to the

Proof of Lemma 9. To prove the lemma, we need the following two facts: There exist a $\lambda_{0}>0$ and $c_{0}, c_{1}, c_{2}>0$ independent of $k$ and $n$ such that

$$
E_{n+1, y}^{h_{N}}\left(\exp \left(\lambda Y_{k}\right) \mid A_{n, l}\right) \leq c_{0}, \text { for } \lambda<\lambda_{0},
$$

and such that

$$
c_{1} \leq E_{n+1, y}^{h_{N}}\left(\hat{Y}_{k} \mid A_{n, l}\right) \leq E_{n+1, y}^{h_{N}}\left(Y_{k} \mid A_{n, l}\right) \leq c_{2} .
$$

So as not to interrupt the flow, we will complete the proof of the lemma and then come back to prove (3.56) and (3.57).

We note that it follows from (3.56) and (3.57) that there exist $C_{1}, C_{2}, C_{3}>0$ independent of $k$ and $n$ such that

$$
P_{n+1, y}^{h_{N}}\left(C_{1} \leq \hat{Y}_{k} \leq C_{2}, Y_{k} \leq C_{2} \mid A_{n, l}\right) \geq C_{3} .
$$

To see this, note that if (3.58) did not hold, then for each positive integer $m$ there would exist a $k_{m}$ and an $n_{m}$ such that $P_{n_{m}+1, y}^{h_{N}}\left(\frac{1}{m} \leq \hat{Y}_{k_{m}} \leq m, Y_{k_{m}} \leq m \mid A_{n_{m}, l}\right) \leq$ $\frac{1}{m}$. It would then follow that

$$
\begin{aligned}
& P_{n_{m}+1, y}^{h_{N}}\left(\hat{Y}_{k_{m}} \leq \frac{1}{m} \mid A_{n_{m}, l}\right)+P_{n_{m}+1, y}^{h_{N}}\left(Y_{k_{m}} \geq m \mid A_{n_{m}, l}\right) \\
& +P_{n_{m}+1, y}^{h_{N}}\left(\hat{Y}_{k_{m}} \geq m \mid A_{n_{m}, l}\right) \geq 1-\frac{1}{m} .
\end{aligned}
$$

However by (3.56), we must have

$$
\lim _{m \rightarrow \infty} P_{n_{m}+1, y}^{h_{N}}\left(Y_{k_{m}} \geq m \mid A_{n_{m}, l}\right)=\lim _{m \rightarrow \infty} P_{n_{m}+1, y}^{h_{N}}\left(\hat{Y}_{k_{m}} \geq m \mid A_{n_{m}, l}\right)=0 .
$$

Using this with (3.59) would then give

$$
\lim _{m \rightarrow \infty} P_{n_{m}+1, y}^{h_{N}}\left(\hat{Y}_{k_{m}} \leq \frac{1}{m} \mid A_{n_{m}, l}\right)=1 .
$$

But then it would follow from the lower bound in (3.57) and from (3.60) that, for any $M>0$,

$$
\liminf _{m \rightarrow \infty} E_{n_{m}+1, y}^{h_{N}}\left(Y_{k_{m}} ; Y_{k_{m}} \geq M \mid A_{n_{m}, l}\right) \geq c_{1} .
$$

From (3.61) and Chebyshev's inequality, we would obtain

$$
\begin{aligned}
& \liminf _{m \rightarrow \infty} E_{n_{m}+1, y}^{h_{N}}\left(\exp \left(\lambda Y_{k_{m}}\right) \mid A_{n_{m}, l}\right) \\
& \geq \frac{1}{M} \exp (\lambda M) \liminf _{m \rightarrow \infty} E_{n_{m}+1, y}^{h_{N}}\left(Y_{k_{m}} ; Y_{k_{m}} \geq M \mid A_{n_{m}, l}\right) \geq \frac{c_{1}}{M} \exp (\lambda M) .
\end{aligned}
$$

Since $M$ is arbitrary, (3.62) contradicts (3.56). This proves (3.58). 
Recalling the independence of the $\left(Y_{j}, \hat{Y}_{j}\right)$ 's under $P_{n+1, y}^{h_{N}}\left(\cdot \mid A_{n, l}\right)$, and letting $Z_{l}=\sum_{\substack{j \in\{1, \ldots, l\} \\ j \neq k}} Y_{j}$, we have

$$
\begin{aligned}
& P_{n+1, y}^{h_{N}}\left(C_{1} \leq \hat{Y}_{k} \leq C_{2}, \sum_{j=0}^{\hat{W}_{n}} Y_{j} \leq K n^{2} \mid A_{n, l}\right) \\
& \geq P_{n+1, y}^{h_{N}}\left(C_{1} \leq \hat{Y}_{k} \leq C_{2}, Y_{k} \leq C_{2} \mid A_{n, l}\right) P_{n+1, y}^{h_{N}}\left(Z_{l} \leq K n^{2}-C_{2} \mid A_{n, l}\right) .
\end{aligned}
$$

A standard large deviations result using (3.56) and (3.57) shows that, for $K$ sufficiently large, there exists a $c>0$ independent of $n$ such that

$$
P_{n+1, y}^{h_{N}}\left(Z_{l} \leq K n^{2}-C_{2} \mid A_{n, l}\right) \geq c, \text { for } l \leq n^{2} .
$$

Now (3.50) follows from (3.58), (3.63) and (3.64).

We now return to prove (3.56) and (3.57). Note that $Y_{k}$ and $\hat{Y}_{k}$ under $P_{n+1, y}^{h_{N}}\left(\cdot \mid A_{n, l}\right)$, are distributed respectively either according to the distributions of $\tau_{m+1}$ and $\int_{0}^{\tau_{m+1}} 1_{G}(r(s)) d s$ under $P_{m, y}^{h_{N}}\left(\cdot \mid \tau_{m+1}<\tau_{m-1} \wedge \sigma, y\left(\tau_{m+1}\right)=\hat{y}\right)$ for some integer $m \geq 2$ and some $y, \hat{y} \in[-1,1]$, or according to the distribution obtained by switching the roles of $m+1$ and $m-1$ above. The calculation we now make holds just as well with the roles of $m+1$ and $m-1$ switched. We claim that there exists a $\lambda_{0}>0$ independent of $m$ such that

$$
E_{m, y}^{h_{N}}\left(\exp \left(\lambda \tau_{m+1}\right) \mid \tau_{m+1}<\tau_{m-1} \wedge \sigma, y\left(\tau_{m+1}\right)=\hat{y}\right) \leq c_{0}, \text { for } \lambda<\lambda_{0} .
$$

Now (3.56) follows from (3.65). In the following parenthetical, we sketch the proof of (3.65), leaving the reader to complete the details. (An argument similar to the one between (3.37) and (3.41) which showed that (3.37) was equivalent to (3.41) shows that (3.65) is equivalent to the same inequality (possibly with different $\lambda_{0}$ and $c_{0}$ ) with the condition $y\left(\tau_{m+1}\right)=\hat{y}$ deleted. Now let $V_{m}(r, y)=P_{r, y}^{h_{N}}\left(\tau_{m+1}<\tau_{m-1} \wedge \sigma\right)$. The $h$-transform of $\frac{1}{2} \Delta^{h_{N}}$ by the function $V_{m}$ is the operator $\frac{1}{2} \Delta^{V_{m} h_{N}}$. To show (3.65) with the condition $y\left(\tau_{m+1}\right)=\hat{y}$ deleted, it is enough to show that the principal eigenvalue for $\frac{1}{2} \Delta^{V_{m} h_{N}}$ in $(m-1, m+1) \times(-1,1)$ with the Neumann boundary condition on $\left(\left(m-1, a_{m-1}\right) \cup\left(b_{m-1}, a_{m}\right) \cup\left(b_{m}, m+1\right)\right) \times\{-1,1\}$ and the Dirichlet boundary condition on $\{m+1\} \times(-1,1)$ is bounded away from zero as a function of $m$. By spectral equivalence under $h$-transforms, this is equivalent to the boundedness away from zero as a function of $m$ for the principal eigenvalue of $\frac{1}{2} \Delta^{h_{N}}$ in $(m-1, m+1) \times(-1,1)$ with the Dirichlet boundary condition on $\{m-1, m+1\} \times(-1,1)$ and on $\left(\left(a_{m-1}, b_{m-1}\right) \cup\left(a_{m}, b_{m}\right)\right) \times\{-1,1\}$, and with the Neumann boundary condition on the rest of the boundary. This last requirement finally can be demonstrated simply using the mini-max technique.)

To show (3.57), we must show that

$$
\left.E_{m, y}^{h_{N}}\left(\int_{0}^{\tau_{m+1}} 1_{G}(r(s)) d s\right) \mid \tau_{m+1}<\tau_{m-1} \wedge \sigma, y\left(\tau_{m+1}\right)=\hat{y}\right) \geq c_{1},
$$

and that

$$
E_{m, y}^{h_{N}}\left(\tau_{m+1} \mid \tau_{m+1}<\tau_{m-1} \wedge \sigma, y\left(\tau_{m+1}\right)=\hat{y}\right) \leq c_{2} .
$$

Again, by the technique used between (3.37) and (3.41), it is enough to show (3.66) and (3.67) with the condition $y\left(\tau_{m+1}\right)=\hat{y}$ deleted. Thus, letting

$$
F_{1}(r, y)=E_{r, y}^{h_{N}}\left(\int_{0}^{\tau_{m+1}} 1_{G}(r(s)) d s \mid \tau_{m+1}<\tau_{m-1} \wedge \sigma\right)
$$


and $F_{2}(r, y)=E_{r, y}^{h_{N}}\left(\tau_{m+1} \mid \tau_{m+1}<\tau_{m-1} \wedge \sigma\right)$, we need to show that

$$
F_{1}(m, y) \geq c_{1} \text { and } F_{2}(m, y) \leq c_{2}
$$

Calculations similar to ones we've already carried out show that

$$
F_{1}(r, y)=\frac{1}{V_{m}(r, y)} E_{r, y}^{h_{N}} \int_{0}^{\tau_{m-1} \wedge \tau_{m+1} \wedge \sigma} V_{m}(r(s)) 1_{G}(r(s)) d s
$$

and

$$
F_{2}(r, y)=\frac{1}{V_{m}(r, y)} E_{r, y}^{h_{N}} \int_{0}^{\tau_{m-1} \wedge \tau_{m+1} \wedge \sigma} V_{m}(r(s)) d s .
$$

Applying Harnack's inequality to $V_{m}$ similar to what we've done before, we conclude from (3.69) and (3.70) that (3.68) will hold as long as

$$
E_{m, y}^{h_{N}} \int_{0}^{\tau_{m-1} \wedge \tau_{m+1} \wedge \sigma} 1_{G}(r(s)) d s \geq c_{1}
$$

and

$$
E_{m, y}^{h_{N}} \tau_{m-1} \wedge \tau_{m+1} \wedge \sigma \leq c_{2}
$$

The bound in (3.72) is very simple while the bound in (3.71), though not difficult, uses stopping times and takes up more space. We leave the details to the reader.

\section{Proof of Lemma 4}

Recall that (1.16) is in effect. By the strong Markov property, we have

$$
\begin{aligned}
& P_{n, y}\left(\sigma<\tau_{n-1} \wedge \tau_{n+1} \mid \tau_{n \pm 1}<\tau_{n \mp 1}\right) \\
& =\frac{E_{n, y}\left(P_{r(\sigma), y(\sigma)}\left(\tau_{n \pm 1}<\tau_{n \mp 1}\right) ; \sigma<\tau_{n-1} \wedge \tau_{n+1}\right)}{P_{n, y}\left(\tau_{n \pm 1}<\tau_{n \mp 1}\right)} .
\end{aligned}
$$

Since $r(\sigma) \in\left(n-\frac{3}{4}, n-\frac{1}{4}\right) \cup\left(n+\frac{1}{4}, n+\frac{3}{4}\right)$, it follows from Harnack's inequality and (4.1) that there exists a constant $c$ independent of $n$ such that

$$
\begin{aligned}
& c P_{n, y}\left(\sigma<\tau_{n-1} \wedge \tau_{n+1}\right) \leq P_{n, y}\left(\sigma<\tau_{n-1} \wedge \tau_{n+1} \mid \tau_{n \pm 1}<\tau_{n \mp 1}\right) \\
& \leq \frac{1}{c} P_{n, y}\left(\sigma<\tau_{n-1} \wedge \tau_{n+1}\right) .
\end{aligned}
$$

The same proof shows that the above inequality also holds with $P_{n, y}^{h_{N}}$ in place of $P_{n, y}$. Thus, in the sequel, we may assume that $\mathcal{P}_{n, y}$ is equal to either $P_{n, y}$ or $P_{n, y}^{h_{N}}$.

To prove the lemma, it will be useful to formulate the problem in an equivalent way for the corresponding unreflected process. Let $\hat{P}_{r, y}$ and $\hat{P}_{r, y}^{h_{N}}$ be the measures obtained by letting the $y$ component of the $P_{r, y}$ and $P_{r, y}^{h_{N}}$ processes run like unreflected Brownian motions on the whole line rather than as reflected ones on $[-1,1]$, and let $\hat{\mathcal{P}}_{r, y}$ be the generic notation for $\hat{P}_{r, y}$ or $\hat{P}_{r, y}^{h_{N}}$. We make the absorption set periodic by defining $\hat{\sigma}=\inf \left\{t \geq 0: r(t) \in \bigcup_{n=1}^{\infty}\left(a_{n}, b_{n}\right), y(t) \in 2 Z+1\right\}$, where $Z$ denotes the integers. Then

$$
\begin{aligned}
\hat{\mathcal{P}}_{r, y}\left(\hat{\sigma}<\tau_{n-1} \wedge \tau_{n+1}\right)=\mathcal{P}_{r, y}\left(\sigma<\tau_{n-1}\right. & \left.\wedge \tau_{n+1}\right), \\
& \quad \text { for }(r, y) \in(n-1, n+1) \times(-1,1) .
\end{aligned}
$$

Thus, it suffices to prove the upper and the lower bound for the left-hand side of $(4.2)$. 
Upper bound. Let $\eta_{j}=\inf \{t \geq 0: y(t)=j\}$. Note that there exists a $c_{0}>0$ such that

$$
\begin{aligned}
\hat{\mathcal{P}}_{r, 2 j}\left(\tau_{n-1} \wedge \tau_{n+1}<\right. & \left.\eta_{2 j-2} \wedge \eta_{2 j+2}\right) \geq c_{0}>0, \\
& \text { for }(r, y) \in\{n\} \times(2 j-1,2 j+1), \\
& \text { or }(r, y) \in(n-1, n+1) \times\{2 j\}, j=0, \pm 1, \ldots .
\end{aligned}
$$

This can be proved easily, for example, by writing

$$
\begin{aligned}
& \left.\hat{\mathcal{P}}_{r, 2 j}\left(\tau_{n-1} \wedge \tau_{n+1}<\eta_{2 j-2} \wedge \eta_{2 j+2}\right) \geq \hat{\mathcal{P}}_{r, 2 j}\left(\tau_{n-1} \wedge \tau_{n+1}<1, \eta_{2 j-2} \wedge \eta_{2 j+2}\right)>1\right) \\
& \quad=\hat{\mathcal{P}}_{r, 2 j}\left(\tau_{n-1} \wedge \tau_{n+1}<1\right) \hat{\mathcal{P}}_{r, 2 j}\left(\eta_{2 j-2} \wedge \eta_{2 j+2}>1\right) .
\end{aligned}
$$

We will show that there exists a $c_{1}>0$ such that

$$
\begin{aligned}
\hat{\mathcal{P}}_{r, y}\left(\hat{\sigma}<\tau_{n-1} \wedge \tau_{n+1}\right. & \left.\wedge \eta_{2 j-2} \wedge \eta_{2 j+2}\right) \leq c_{1}\left(\frac{1}{\log \rho_{n-1}}+\frac{1}{\log \rho_{n}}\right), \\
& \text { for }(r, y) \in\{n\} \times(2 j-2,2 j+2), \\
& \text { or }(r, y) \in(n-1, n+1) \times\{2 j\}, j=0, \pm 1, \ldots .
\end{aligned}
$$

We now complete the proof of the upper bound using (4.3) and (4.4), and then return to prove (4.4). Of course it's enough to prove the bound for $\rho_{n}$ small. We argue as follows. Assume that $\rho_{n}$ is sufficiently small so that the right-hand side of (4.4) is less than one half the value $c_{0}$ appearing in the right-hand side of (4.3). Starting from $(n, y)$ with $y \in(-1,1)$, we wait until time $\hat{\sigma} \wedge \tau_{n-1} \wedge \tau_{n+1} \wedge \eta_{2} \wedge$ $\eta_{-2}$. The probability that this time is in fact equal to $\hat{\sigma}$, in which case the event $\left\{\hat{\sigma}<\tau_{n-1} \wedge \tau_{n+1}\right\}$ occurs, is no more than the right-hand side of (4.4). The probability that this time is equal to $\tau_{n-1} \wedge \tau_{n+1}$, in which case the event $\{\hat{\sigma}<$ $\left.\tau_{n-1} \wedge \tau_{n+1}\right\}$ does not occur, is at least $\frac{1}{2} c_{0}$. If neither of these two cases occurs, then the above time is equal to $\eta_{2} \wedge \eta_{-2}$, in which case one does not yet know whether the event $\left\{\hat{\sigma}<\tau_{n-1} \wedge \tau_{n+1}\right\}$ will occur. Say, for example, that $\eta_{2} \wedge \eta_{-2}=\eta_{2}$. Then starting anew from $\left(r\left(\eta_{2}\right), y\left(\eta_{2}\right)\right)=\left(r\left(\eta_{2}\right), 2\right)$, with $r\left(\eta_{2}\right) \in(n-1, n+1)$, we wait until time $\hat{\sigma} \wedge \tau_{n-1} \wedge \tau_{n+1} \wedge \eta_{0} \wedge \eta_{4}$. The probability that this time is equal to $\hat{\sigma}$, in which case the event $\hat{\sigma}<\tau_{n-1} \wedge \tau_{n+1}$ occurs, is no more than the right-hand side of (4.4), while the probability that it is equal to $\tau_{n-1} \wedge \tau_{n+1}$, in which case the event $\hat{\sigma}<\tau_{n-1} \wedge \tau_{n+1}$ does not occur, is at least $\frac{1}{2} c_{0}$. If neither of these two cases occurs, then the above time is equal to $\sigma_{4} \wedge \sigma_{0}$ and one does not yet know whether the event $\hat{\sigma}<\tau_{n-1} \tau_{n+1}$ will occur. Continuing like this, it follows that

$$
\begin{aligned}
\hat{\mathcal{P}}_{n, y}\left(\hat{\sigma}<\tau_{n-1} \wedge \tau_{n+1}\right)<c_{1}\left(\frac{1}{\left|\log \rho_{n}\right|}+\frac{1}{\left|\log \rho_{n-1}\right|}\right) \sum_{j=0}^{\infty}\left(1-\frac{1}{2} c_{0}\right)^{j} \\
=\frac{2}{c_{0}} c_{1}\left(\frac{1}{\left|\log \rho_{n}\right|}+\frac{1}{\left|\log \rho_{n-1}\right|}\right),
\end{aligned}
$$

which gives the upper bound.

We now return to prove (4.4). Suppressing the dependence on $n$ and $j$, let $B_{1}$ denote the disk of radius $\frac{1}{2} \rho_{n}=\frac{1}{2}\left(b_{n}-a_{n}\right)$ centered at $\left(r_{1}, y_{1}\right) \equiv\left(\frac{a_{n}+b_{n}}{2}, 2 j+1\right)$, which is the midpoint of the line segment $\left(a_{n}, b_{n}\right) \times\{2 j+1\}$. Define similarly

$B_{2}$ with center $\left(r_{2}, y_{2}\right)=\left(\frac{a_{n}+b_{n}}{2}, 2 j-1\right)$ and radius $\frac{1}{2} \rho_{n}$,

$B_{3}$ with center $\left(r_{3}, y_{3}\right)=\left(\frac{a_{n-1}+b_{n-1}}{2}, 2 j+1\right)$ and radius $\frac{1}{2} \rho_{n-1}$, and

$B_{4}$ with center $\left(r_{4}, y_{4}\right)=\left(\frac{a_{n-1}+b_{n-1}}{2}, 2 j-1\right)$ and radius $\frac{1}{2} \rho_{n-1}$. Again suppressing the dependence on $n$ and $j$, let $S_{i}$, for $i=1, \ldots, 4$, be the disk of radius 4 
centered at $\left(r_{i}, y_{i}\right)$. Note that $(r, y) \notin \bigcup_{i=1}^{4} B_{i}$, if $(r, y)$ is as specified in (4.4), and note also that $(n-1, n+1) \times(2 j-2,2 j+2) \subset S_{i}, i=1, \ldots, 4$. Let $\tau_{B_{i}}=\inf \{t \geq 0$ : $\left.(r(t), y(t)) \in B_{i}\right\}, i=1, \ldots, 4$, and let $T_{S_{i}}=\inf \left\{t \geq 0:(r(t), y(t)) \notin S_{i}\right\}, i=1, \ldots, 4$. Then under $\hat{\mathcal{P}}_{r, y}$, with $(r, y)$ as specified in (4.4), we have

$$
\hat{\sigma} \geq \min _{i=1, \ldots, 4} \tau_{B_{i}} \text { a.s. }
$$

and

$$
\tau_{n-1} \wedge \tau_{n+1} \wedge \eta_{2 j-2} \wedge \eta_{2 j+2}<T_{S_{i}} \text { a.s., for } i=1, \ldots, 4 \text {. }
$$

Thus,

$$
\hat{\mathcal{P}}_{r, y}\left(\hat{\sigma}<\tau_{n-1} \wedge \tau_{n+1} \wedge \eta_{2 j-2} \wedge \eta_{2 j+2}\right) \leq \sum_{i=1}^{4} \hat{\mathcal{P}}_{r, y}\left(\tau_{B_{i}}<T_{S_{i}}\right),
$$

for $(r, y)$ as specified in (4.4).

We now make an argument which will allow us to bound from above the expression on the right-hand side of (4.5) by a constant times the corresponding expression obtained by replacing the probabilities appearing there by standard Wiener measure. Let $s=\left(\left(r-r_{1}\right)^{2}+\left(y-y_{1}\right)^{2}\right)^{\frac{1}{2}}$ and $\phi=\arctan \frac{y-y_{1}}{r-r_{1}}$ denote polar coordinates for $(r, y)$, centered at $\left(r_{1}, y_{1}\right)$. Noting that the distance between $(r, y)$ as specified in (4.4) and $\bigcup_{i=1}^{4} B_{i}$ is at least $\frac{1}{4}$ and that the distance from $(r, y)$ as specified in (4.4) and $\bigcup_{i=1}^{4} S_{i}^{c}$ is at least 1 , it follows that

$$
\hat{\mathcal{P}}_{r, y}\left(\tau_{B_{1}}<T_{S_{1}}\right)=\tilde{\mathcal{P}}_{s, \phi}^{\left(b_{s}, b_{\phi}\right)}\left(\tilde{\tau}_{\frac{1}{2} \rho_{n}}<\tilde{\tau}_{4}\right),
$$

where $\tilde{\mathcal{P}}_{s, \phi}^{\left(b_{s}, b_{\phi}\right)}$ is the measure corresponding to an operator of the form

$$
\left(\frac{1}{2} \frac{\partial^{2}}{\partial s^{2}}+\frac{1}{2 s} \frac{\partial}{\partial s}+\frac{1}{2 s^{2}} \frac{\partial^{2}}{\partial \phi^{2}}\right)+\left(b_{s}(r, y) \frac{\partial}{\partial s}++\frac{b_{\phi}(r, y)}{s^{2}} \frac{\partial}{\partial \phi}\right)
$$

with $\left|b_{s}\right|$ and $\left|b_{\phi}\right|$ bounded independent of $n$ and $j, \tilde{\tau}_{s}=\inf \{t \geq 0: s(t)=s\}$, and $s=\left(r^{2}+y^{2}\right)^{\frac{1}{2}} \in\left(\frac{1}{4}, 3\right)$. Of course, if we let $s, \phi$ denote polar coordinates centered at $\left(r_{i}, y_{i}\right), i=2,3,4$, then (4.6) also holds with $B_{1}$ replaced by $B_{2}$, or with $B_{1}$ replaced by $B_{3}$ or $B_{4}$ and $\rho_{n}$ replaced by $\rho_{n-1}$. Let $M>0$ be an upper bound on $\left|b_{s}\right|$. By comparison, it follows that

$$
\tilde{\mathcal{P}}_{s, \phi}^{\left(b_{s}, b_{\phi}\right)}\left(\tilde{\tau}_{\frac{1}{2} \rho_{n}}<\tilde{\tau}_{4}\right) \leq \tilde{\mathcal{P}}_{s, \phi}^{(-M, 0)}\left(\tilde{\tau}_{\frac{1}{2} \rho_{n}}<\tilde{\tau}_{4}\right) .
$$

In the notation we are using, $\tilde{\mathcal{P}}_{s, \phi}^{(0,0)}$ is standard two-dimensional Wiener measure, and by the Cameron-Martin formula, we have

$$
\begin{aligned}
& \tilde{\mathcal{P}}_{s, \phi}^{(-M, 0)}\left(\tilde{\tau}_{\frac{1}{2} \rho_{n}}<\tilde{\tau}_{4}\right)=\tilde{\mathcal{E}}_{s, \phi}^{(0,0)}\left(\exp \left(M\left(s-\frac{1}{2} \rho_{n}\right)-\frac{M^{2}}{2} \tilde{\tau}_{\frac{1}{2} \rho_{n}}\right) ; \tilde{\tau}_{\frac{1}{2} \rho_{n}}<\tilde{\tau}_{4}\right) \\
& \leq c \tilde{\mathcal{P}}_{s, \phi}^{0,0}\left(\tilde{\tau}_{\frac{1}{2} \rho_{n}}<\tilde{\tau}_{4}\right) .
\end{aligned}
$$

For two-dimensional Brownian motion, we have the well-known formula

$$
\tilde{\mathcal{P}}_{s, \phi}^{(0,0)}\left(\tilde{\tau}_{\frac{1}{2} \rho_{n}}<\tilde{\tau}_{4}\right)=\frac{\log s-\log 4}{\log \left(\frac{1}{2} \rho_{n}\right)-\log 4}, \text { for } s=\left(r^{2}+y^{2}\right)^{\frac{1}{2}} \in\left(\frac{1}{2} \rho_{n}, 4\right) .
$$

Now (4.4) follows from (4.5)-(4.9).

Lower bound. Consider only those $n$ large enough so that $\rho_{n}<\frac{1}{5}$. Let $\left(r_{0}, y_{0}\right)=$ $\left(\frac{a_{n}+b_{n}}{2}, 1\right)$ and let $D_{i}, i=1, \ldots, 4$, be the nested sequence of disks centered at $\left(r_{0}, y_{0}\right)$ 
with radii $\frac{1}{4} \rho_{n}, \frac{1}{2} \rho_{n}, \frac{1}{10}$, and $\frac{1}{5}$ respectively. (We suppress the dependence of $D_{i}$ on $n$.) Note that by $(1.16), D_{4} \subset(n, n+1) \times R$ and

$$
\inf _{n} \operatorname{dist}\left\{\{n\} \times(-1,1), D_{3}\right\}>0 .
$$

Let $\tau_{D_{i}}=\inf \left\{t \geq 0:(r(t), y(t)) \in D_{i}\right\}$ and let $T_{D_{i}}=\inf \left\{t \geq 0:(r(t), y(t)) \notin D_{i}\right\}$. A standard argument using (4.10) and the uniform boundedness of the coefficients shows that

$$
\hat{\mathcal{P}}_{n, y}\left(\tau_{D_{3}}<\tau_{n-1} \wedge \tau_{n+1}\right) \geq c>0, \text { for } y \in(-1,1) \text { and all } n .
$$

We will show that there exists a $c>0$ such that

$$
\hat{\mathcal{P}}_{r, y}\left(\tau_{D_{1}}<T_{D_{4}}\right) \geq \frac{c}{\left|\log \rho_{n}\right|}, \text { for }(r, y) \in \partial D_{3} \text { and all } n,
$$

and that

$$
\hat{\mathcal{P}}_{r, y}\left(\hat{\sigma}<T_{D_{2}}\right) \geq c>0, \text { for }(r, y) \in \partial D_{1} \text { and all } n .
$$

The lower bound now follows from the strong Markov property and (4.11)-(4.13). It remains to prove (4.12) and (4.13).

We begin with (4.12). Making the same type of argument used in (4.6) and (4.7), and using the same notation, we have

$$
\hat{\mathcal{P}}_{r, y}\left(\tau_{D_{1}}<T_{D_{4}}\right) \geq \tilde{\mathcal{P}}_{\frac{1}{10}, \phi}^{(M, 0)}\left(\tilde{\tau}_{\frac{1}{4} \rho_{n}}<\tilde{\tau}_{\frac{1}{5}}\right), \text { for }(r, y) \in \partial D_{3} .
$$

Using the Cameron-Martin formula as we did in (4.8) gives

$$
\tilde{\mathcal{P}}_{\frac{1}{10}, \phi}^{(M, 0)}\left(\tilde{\tau}_{\frac{1}{4} \rho_{n}}<\tilde{\tau}_{\frac{1}{5}}\right)=\tilde{\mathcal{E}}_{\frac{1}{10}, \phi}^{(0,0)}\left(\exp \left(M\left(\frac{1}{4} \rho_{n}-\frac{1}{10}\right)-\frac{M^{2}}{2} \tilde{\tau}_{\frac{1}{4} \rho_{n}}\right) ; \tilde{\tau}_{\frac{1}{4} \rho_{n}}<\tilde{\tau}_{\frac{1}{5}}\right) .
$$

Recall that $\tilde{\mathcal{P}}_{s, \phi}^{(0,0)}$ is standard two-dimensional Wiener measure. Let

$$
h(s) \equiv \tilde{\mathcal{P}}_{s, \phi}^{(0,0)}\left(\tilde{\tau}_{\frac{1}{4} \rho_{n}}<\tilde{\tau}_{\frac{1}{5}}\right)=\frac{\log s-\log \frac{1}{5}}{\log \frac{\rho_{n}}{4}-\log \frac{1}{5}} .
$$

Then the the conditioned process corresponding to $\tilde{\mathcal{P}}_{\frac{1}{10}, \phi}^{(0,0)}\left(\cdot \mid \tilde{\tau}_{\frac{1}{4} \rho_{n}}<\tilde{\tau}_{\frac{1}{5}}\right)$ is generated by the $h$-transformed operator

$$
\left(\frac{1}{2} \frac{\partial^{2}}{\partial s^{2}}+\frac{1}{2 s} \frac{\partial}{\partial s}+\frac{1}{2 s^{2}} \frac{\partial^{2}}{\partial \phi^{2}}\right)+\frac{h^{\prime}}{h} \frac{\partial}{\partial s} .
$$

The function $u_{n}(s) \equiv \tilde{\mathcal{E}}_{s, \phi}^{(0,0)}\left(\tilde{\tau}_{\frac{1}{4} \rho_{n}} \mid \tilde{\tau}_{\frac{1}{4} \rho_{n}}<\tilde{\tau}_{\frac{1}{5}}\right)$ is the minimal positive solution to

$$
\frac{1}{2} u_{n}^{\prime \prime}+\frac{1}{2 s} u_{n}^{\prime}+\frac{h^{\prime}}{h} u_{n}^{\prime}=-1, \text { for } s \in\left(\frac{1}{4} \rho_{n}, \frac{1}{5}\right) .
$$

Solving, we obtain

$$
\begin{aligned}
& u_{n}(s)=\int_{\frac{1}{4} \rho_{n}}^{s} \frac{1}{t h^{2}(t)}\left(\int_{t}^{\frac{1}{5}} 2 r h^{2}(r) d r\right) d t \\
& =\int_{\frac{1}{4} \rho_{n}}^{s} \frac{1}{t\left(\log t-\log \frac{1}{5}\right)^{2}}\left(\int_{t}^{\frac{1}{5}} 2 r\left(\log r-\log \frac{1}{5}\right)^{2} d r\right) d t .
\end{aligned}
$$

One can check that the integrand on the right hand side of (4.17) is integrable at 0 ; therefore we can conclude that

$$
\sup _{n} \tilde{\mathcal{E}}_{\frac{1}{10}, \phi}^{(0,0)}\left(\tilde{\tau}_{\frac{1}{4} \rho_{n}} \mid \tilde{\tau}_{\frac{1}{4} \rho_{n}}<\tilde{\tau}_{\frac{1}{5}}\right)<\infty .
$$


From (4.18), we conclude that there exists an $L$ such that

$$
\sup _{n} \tilde{\mathcal{P}}_{\frac{1}{10}, \phi}^{(0,0)}\left(\tilde{\tau}_{\frac{1}{4} \rho_{n}} \leq L \mid \tilde{\tau}_{\frac{1}{4} \rho_{n}}<\tilde{\tau}_{\frac{1}{5}}\right)>\frac{1}{2} .
$$

From (4.19) and (4.15) we obtain

$$
\begin{aligned}
& \tilde{\mathcal{P}}_{\frac{1}{10}, \phi}^{(M, 0)}\left(\tilde{\tau}_{\frac{1}{4} \rho_{n}}<\tilde{\tau}_{\frac{1}{5}}\right) \\
& =\tilde{\mathcal{E}}_{\frac{1}{10}, \phi}^{(0,0)}\left(\exp \left(M\left(\frac{1}{4} \rho_{n}-\frac{1}{10}\right)-\frac{M^{2}}{2} \tilde{\tau}_{\frac{1}{4} \rho_{n}}\right) \mid \tilde{\tau}_{\frac{1}{4} \rho_{n}}<\tilde{\tau}_{\frac{1}{5}}\right) \tilde{\mathcal{P}}_{\frac{1}{10}, \phi}^{(0,0)}\left(\tilde{\tau}_{\frac{1}{4} \rho_{n}}<\tilde{\tau}_{\frac{1}{5}}\right) \\
& \geq \frac{1}{2} \exp \left(-\frac{1}{10} M-\frac{M^{2} L}{2}\right) P_{\frac{1}{10}, \phi}^{(0,0)}\left(\tilde{\tau}_{\frac{1}{4} \rho_{n}}<\tilde{\tau}_{\frac{1}{5}}\right) .
\end{aligned}
$$

Now (4.12) follows from (4.14), (4.20) and (4.16).

We now prove (4.13). Let $P^{W}$ denote standard two-dimensional Wiener measure for $(r(t), y(t))$. Let $b(r)$ equal either $\frac{d-1}{2 r}$ or $\frac{3-d}{2 r}$ depending on whether $\hat{\mathcal{P}}_{r, y}$ is representing $\hat{P}_{r, y}$ or $\hat{P}_{r, y}^{h_{N}}$, and let $B$ be a primitive of $b$. Using the Cameron-Martin formula, we have

$$
\begin{aligned}
& \hat{\mathcal{P}}_{r, y}\left(\hat{\sigma}<T_{D_{2}}\right) \geq \hat{\mathcal{P}}_{r, y}\left(\hat{\sigma}<1 \wedge T_{D_{2}}\right) \\
& =E_{r, y}^{W}\left(\exp \left(\int_{0}^{\hat{\sigma}} b(r(t)) d r(t)-\frac{1}{2} \int_{0}^{\hat{\sigma}} b^{2}(r(t)) d t\right) ; \hat{\sigma}<1 \wedge T_{D_{2}}\right) \\
& =E_{r, y}^{W}\left(\exp \left(B(r(\hat{\sigma}))-B(r)-\frac{1}{2} \int_{0}^{\hat{\sigma}} b^{\prime}(r(t)) d t-\frac{1}{2} \int_{0}^{\hat{\sigma}} b^{2}(r(t)) d t\right) ; \hat{\sigma}<1 \wedge T_{D_{2}}\right) \\
& \geq c P_{r, y}^{W}\left(\hat{\sigma}<1 \wedge T_{D_{2}}\right), \text { for } r \geq 1, \text { and all } y, n,
\end{aligned}
$$

where $c>0$. By Brownian scaling, there exists a $c>0$ that

$$
P_{r, y}^{W}\left(\hat{\sigma}<1 \wedge T_{D_{2}}\right) \geq c, \text { for }(r, y) \in \partial D_{1} \text { and all } n .
$$

Now (4.13) follows from (4.21) and (4.22).

\section{REFERENCES}

1. Ioffe, D. and Pinsky, R., Positive harmonic functions vanishing on the boundary for the Laplacian in unbounded horn-shaped domains, Trans. Amer. Math. Soc. 342 (1994), 773-791. MR 94h:60114

2. Pinsky, R., A new approach to the Martin boundary via diffusions conditioned to hit a compact set, Annals of Probab. 21 (1993), 453-481. MR 94f:60098

3. Pinsky, R., Positive Harmonic Functions and Diffusion, Cambridge Univ. Press, 1995. MR 96m:60179

Department of Mathematics, Technion-Israel Institute of Mathematics, Haifa 32000, ISRAEL

E-mail address: pinsky@techunix.technion.ac.il 